\title{
Hypersingular formulation for boundary stress evaluation revisited. Part 2: Corners.
}

\author{
Alberto Salvadori \\ DICATA, University of Brescia, via Branze 43, 25123 Brescia, Italy
}

\begin{abstract}
This work focuses on the use of the hypersingular boundary integral equation to evaluate the linear elastic stress tensor on the boundary. A companion paper [1] has been devoted to smooth boundaries: in the present work, the traction equation is analyzed at a corner, in the presence of a Lipschitz boundary. Properties of the hypersingular and of the strongly singular operator are discussed together with properties of the whole linear elastic integral operator.
\end{abstract}

\section{Keywords}

Hypersingular boundary integral equations; stress evaluation; free terms; boundary element method

\section{Introduction}

In the first part of this work [1], the hypersingular integral formulation of the linear elastic problem has been revised in view of its use in evaluating the stress tensor on a smooth boundary. There, "smooth" stood for every boundary for which a normal and a tangent vector to the boundary made sense. This second part aims at analyzing the hypersingular identity

$$
\begin{aligned}
\chi_{\Omega}(\mathbf{x}) & \mathbf{p}(\mathbf{x})+ \\
& \int_{\Gamma_{p}} \mathbf{G}_{p p}(\mathbf{r} ; \mathbf{n}(\mathbf{x}) ; \mathbf{l}(\mathbf{y})) \mathbf{u}(\mathbf{y}) \mathrm{d} \Gamma_{\mathbf{y}}+\int_{\Gamma_{u}} \mathbf{G}_{p p}(\mathbf{r} ; \mathbf{n}(\mathbf{x}) ; \mathbf{l}(\mathbf{y})) \overline{\mathbf{u}}(\mathbf{y}) \mathrm{d} \Gamma_{\mathbf{y}}= \\
& \int_{\Gamma_{u}} \mathbf{G}_{p u}(\mathbf{r} ; \mathbf{n}(\mathbf{x})) \mathbf{p}(\mathbf{y}) \mathrm{d} \Gamma_{\mathbf{y}}+\int_{\Gamma_{p}} \mathbf{G}_{p u}(\mathbf{r} ; \mathbf{n}(\mathbf{x})) \overline{\mathbf{p}}(\mathbf{y}) \mathrm{d} \Gamma_{\mathbf{y}}, \quad \mathbf{x} \notin \Gamma
\end{aligned}
$$

and the traction boundary integral equation (BIE),

$$
\begin{aligned}
& \mathbf{D}(\mathbf{x}) \mathbf{p}(\mathbf{x})+ \\
& \quad f_{\Gamma_{p}} \mathbf{G}_{p p}(\mathbf{r} ; \mathbf{n}(\mathbf{x}) ; \mathbf{l}(\mathbf{y})) \mathbf{u}(\mathbf{y}) \mathrm{d} \Gamma_{\mathbf{y}}+f_{\Gamma_{u}} \mathbf{G}_{p p}(\mathbf{r} ; \mathbf{n}(\mathbf{x}) ; \mathbf{l}(\mathbf{y})) \overline{\mathbf{u}}(\mathbf{y}) \mathrm{d} \Gamma_{\mathbf{y}}=
\end{aligned}
$$




$$
f_{\Gamma_{u}} \mathbf{G}_{p u}(\mathbf{r} ; \mathbf{n}(\mathbf{x})) \mathbf{p}(\mathbf{y}) \mathrm{d} \Gamma_{\mathbf{y}}+f_{\Gamma_{p}} \mathbf{G}_{p u}(\mathbf{r} ; \mathbf{n}(\mathbf{x})) \overline{\mathbf{p}}(\mathbf{y}) \mathrm{d} \Gamma_{\mathbf{y}}, \quad \mathbf{x} \in \Gamma
$$

on boundaries that present corners. In dealing with smooth boundaries, $\mathbf{p}(\mathbf{x})$ in equation (2) denotes the traction at $\mathbf{x} \in \Gamma$ referring to the outward normal $\mathbf{l}(\mathbf{x})$, that is $\mathbf{p}(\mathbf{x})$ stands for $\mathbf{p}(\mathbf{x}, \mathbf{l}(\mathbf{x}))$. Proposition 4.1 in reference [1] states that equation (2) holds even with respect to any direction $\mathbf{n}(\mathbf{x})$. The present work aims at extending such a result in the presence of corners, when normal at the boundary is not uniquely defined.

Similarly to [1], the contribution of the hypersingular kernel and of the strongly singular kernel to the free term $\mathbf{D}$ are separately considered. To this aim, the open domain $\Omega \in \mathbf{R}^{2}$, and its boundary $\Gamma$, formed by a finite number of curve elements, are treated as follows.

Take $\mathbf{x}_{0} \in \Gamma, I_{\varepsilon} \subset \mathbf{R}^{2}$ a neighborhood of it, and define $\Gamma_{\varepsilon} \stackrel{\text { def }}{=} \Gamma \bigcap I_{\varepsilon}$ (see figure 1-a). Let $\tilde{\mathbf{y}}(s)=\left\{\tilde{y}_{1}(s), \tilde{y}_{2}(s)\right\}, 0 \leq s \leq l$ be the parametric equations of curve $\Gamma_{\varepsilon}$ with $s$ denoting the curvilinear abscissa; assume $\tilde{\mathbf{y}}(s)$ differentiable in $0 \leq s \leq l$ with the exception of a single point $s_{0}$; at $s_{0}$ assume that they exist $\lim _{s \rightarrow s_{0}^{+}} \tilde{\mathbf{y}}^{\prime}(s)$ and $\lim _{s \rightarrow s_{0}^{-}} \tilde{\mathbf{y}}^{\prime}(s)$. Define $\Gamma_{\varepsilon}^{-} \subset \Gamma_{\varepsilon}$ the curve parameterized by $\tilde{\mathbf{y}}(s)=\left\{\tilde{y}_{1}(s), \tilde{y}_{2}(s)\right\}, 0 \leq s \leq s_{0}$ and $\Gamma_{\varepsilon}^{+} \stackrel{\text { def }}{=} \Gamma_{\varepsilon}-\Gamma_{\varepsilon}^{-}$its complementary part described by $\tilde{\mathbf{y}}(s)=\left\{\tilde{y}_{1}(s), \tilde{y}_{2}(s)\right\}, s_{0} \leq s \leq l$. Consider two local orthogonal coordinate systems $\mathcal{L}^{+}$and $\mathcal{L}^{-}$centered at $\mathbf{x}_{0}=\tilde{\mathbf{y}}\left(s_{0}\right) \in \Gamma_{\varepsilon}$, as in figure 1b. Axis $y_{1}, y_{2}$ are taken as tangent and normal to $\Gamma_{\varepsilon}$ at $\mathbf{x}_{0}$, respectively, in a limit sense $s \rightarrow s_{0}^{+}$and $s \rightarrow s_{0}^{-}$. Point $\mathbf{x}_{0}$ is selected such that the parametric equations of $\Gamma_{\varepsilon} \operatorname{read} \mathbf{y}\left(y_{1}^{+}\right)=\left\{y_{1}^{+}, y_{2}^{+}\left(y_{1}^{+}\right)\right\}, y_{1}^{+} \in I_{\varepsilon}^{+} \stackrel{\text { def }}{=}[0, \varepsilon]$ in $\mathcal{L}^{+}$and $\mathbf{y}\left(y_{1}^{-}\right)=$ $\left\{y_{1}^{-}, y_{2}^{-}\left(y_{1}^{-}\right)\right\}, y_{1}^{-} \in I_{\varepsilon}^{-} \stackrel{\text { def }}{=}[-\varepsilon, 0]$ in $\mathcal{L}^{-}$.

Split equation (1) on $\Gamma$ in $\Gamma_{\varepsilon}$ and its complementary part. Free terms come out from integrals

$$
\begin{array}{lr}
\int_{\Gamma_{\varepsilon}} \mathbf{G}_{p p}(\mathbf{r} ; \mathbf{n}(\mathbf{x}) ; \mathbf{l}(\mathbf{y})) \mathbf{u}(\mathbf{y}) \mathrm{d} \Gamma_{\mathbf{y}} & \Omega \ni \mathbf{x} \rightarrow \mathbf{x}_{0} \in \Gamma_{\varepsilon} \\
\int_{\Gamma_{\varepsilon}} \mathbf{G}_{p u}(\mathbf{r} ; \mathbf{n}(\mathbf{x})) \mathbf{p}(\mathbf{y}) \mathrm{d} \Gamma_{\mathbf{y}} & \Omega \ni \mathbf{x} \rightarrow \mathbf{x}_{0} \in \Gamma_{\varepsilon}
\end{array}
$$

the singular part of which will be evaluated analytically by imposing suitable regularity to the solution.

Suppose $\mathbf{u} \in C^{1, \alpha}\left(\Gamma_{\varepsilon}\right)$, allowing an asymptotic analysis of integral (3), by expanding $\mathbf{u}(\mathbf{y})$ around $\mathbf{x}_{0}$ as follows:

$$
\mathbf{u}(\mathbf{y})=\mathbf{u}\left(\mathbf{x}_{0}\right)+\left.\mathbf{J A C}_{\mathbf{y}}(\mathbf{u})\right|_{\mathbf{x}_{0}}\left(\mathbf{y}-\mathbf{x}_{0}\right)+O\left(\left\|\mathbf{y}-\mathbf{x}_{0}\right\|^{1+\alpha}\right)
$$

for any $0<\alpha \leq 1$. The Jacobian term is linked to the strain tensor $\epsilon$ by the decomposition:

$$
\left.\mathbf{J A C}_{\mathbf{y}}(\mathbf{u})\right|_{\mathbf{x}_{0}}=\boldsymbol{\epsilon}\left(\mathbf{x}_{0}\right)+\operatorname{SKW}\left(\left.\mathbf{J A C}_{\mathbf{y}}(\mathbf{u})\right|_{\mathbf{x}_{0}}\right)
$$




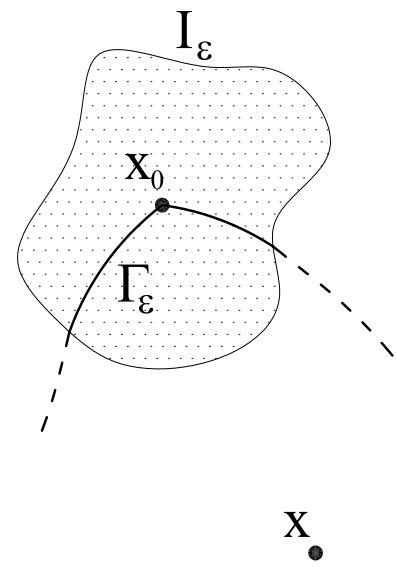

a) Boundary $\Gamma_{\varepsilon}$ and its complementary part.

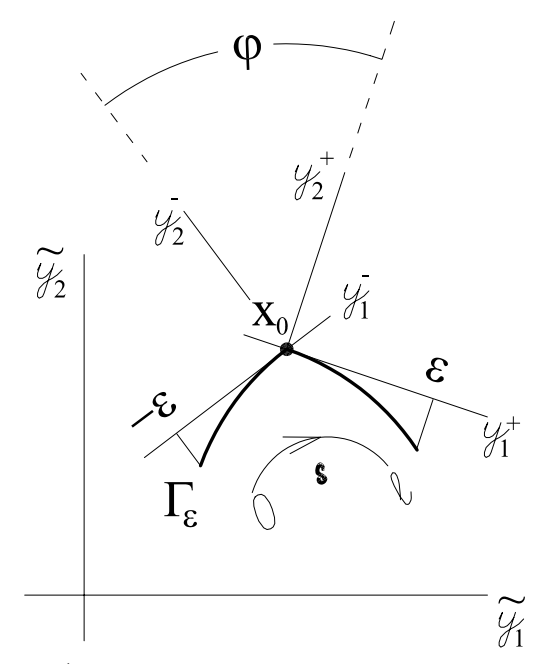

b) Local and global references on $\Gamma_{\varepsilon}$

Figure 1: Description of $\Gamma_{\varepsilon}$.

By assuming that the skew-symmetric part, which describes only rigid body modes, vanishes, the integral (3) becomes:

$$
\begin{aligned}
& \int_{\Gamma_{\varepsilon}} \mathbf{G}_{p p}()\left[\mathbf{u}(\mathbf{y})-\mathbf{u}\left(\mathbf{x}_{0}\right)-\boldsymbol{\epsilon}\left(\mathbf{x}_{0}\right)\left(\mathbf{y}-\mathbf{x}_{0}\right)\right] \mathrm{d} \Gamma+ \\
& +\int_{\Gamma_{\varepsilon}} \mathbf{G}_{p p}() \mathrm{d} \Gamma \mathbf{u}\left(\mathbf{x}_{0}\right)+\int_{\Gamma_{\varepsilon}} \mathbf{G}_{p p}() \boldsymbol{\epsilon}\left(\mathbf{x}_{0}\right) \mathbf{y} \mathrm{d} \Gamma \quad \Omega \ni \mathbf{x} \rightarrow \mathbf{x}_{0} \in \Gamma_{\varepsilon}
\end{aligned}
$$

In view of eq. (5) and of the hyper singularity of the kernel $\mathbf{G}_{p p}$, the function

$$
\mathbf{G}_{p p}(\mathbf{x}-\mathbf{y} ; \mathbf{n}(\mathbf{x}) ; \mathbf{l}(\mathbf{y}))\left[\mathbf{u}(\mathbf{y})-\mathbf{u}\left(\mathbf{x}_{0}\right)-\boldsymbol{\epsilon}\left(\mathbf{x}_{0}\right)\left(\mathbf{y}-\mathbf{x}_{0}\right)\right]
$$

is Lebesgue integrable on $\Gamma_{\epsilon}$ also when $\mathbf{x} \in \Omega$ moves to $\mathbf{x}_{0} \in \Gamma_{\varepsilon}$. Free terms arise therefore from the remaining terms, which will be dealt with in Section 2 by means of recently proposed formulae [2]. As a main result, it will be shown that the presence of a corner causes the non-existence of limit (7): it turns out to consist of the finite part of Hadamard (HFP), of a bounded term independent on the size of the support (expected to be the free term contribution $\mathbf{D}_{p p}(\mathbf{x}, \mathbf{n}(\mathbf{x})$ )), and of an unbounded logarithmic term. This last term is proved to vanish on every $C^{1}$ boundary, therefore it is merely due to the presence of the corner. On the other hand, even the "candidate" to be the free term has a major problem: it shows to depend on the direction by which the limit is taken, and cannot be defined by a limit process. It seems in conclusion that the property of convergence of the hypersingular integral to a HFP plus a free term, proved on smooth domains, 
cannot be extended to corners despite some terms that are present "in nuce". It should be questionable, accordingly, if equation (2) may be written at a corner.

Assume $\mathbf{p} \in C^{0, \alpha}\left(\Gamma_{\varepsilon}\right)$. This allows to expand $\mathbf{p}(\mathbf{y})$ around $\mathbf{x}_{0}$ as follows:

$$
\mathbf{p}(\mathbf{y})=\mathbf{p}\left(\mathbf{x}_{0}\right)+O\left(\left\|\mathbf{y}-\mathbf{x}_{0}\right\|^{\alpha}\right)
$$

for any $0<\alpha \leq 1$ and integral (4) becomes:

$$
\int_{\Gamma_{\varepsilon}} \mathbf{G}_{p u}()\left[\mathbf{p}(\mathbf{y})-\mathbf{p}\left(\mathbf{x}_{0}\right)\right] \mathrm{d} \Gamma+\int_{\Gamma_{\varepsilon}} \mathbf{G}_{p u}() \mathrm{d} \Gamma \mathbf{p}\left(\mathbf{x}_{0}\right) \quad \Omega \ni \mathbf{x} \rightarrow \mathbf{x}_{0} \in \Gamma_{\varepsilon}
$$

In view of eq. (8) and of the strong singularity of kernel $\mathbf{G}_{p u}$, function

$$
\mathbf{G}_{p u}(\mathbf{x}-\mathbf{y} ; \mathbf{n}(\mathbf{x}))\left[\mathbf{p}(\mathbf{y})-\mathbf{p}\left(\mathbf{x}_{0}\right)\right]
$$

is integrable on $\Gamma_{\epsilon}$ also when $\mathbf{x} \in \Gamma_{\varepsilon}$. Free terms arise therefore from the remaining term, which will be considered in Section 3. The limit behavior of the strongly singular integral is similar to the hypersingular one, showing Cauchy's principal value $(\mathrm{CPV}$ ), an unbounded logarithmic term, and a bounded factor (which in nuce is the free term contribution $\mathbf{D}_{p u}(\mathbf{x}, \mathbf{n}(\mathbf{x}))$ ) that cannot be defined by a limit process.

In section 4 it will be proved that a matrix $\mathbf{D}$ exists such that equation (2) holds at a corner. Such a matrix is well defined by a limit process and is a linear combination of the "candidate" free terms $\mathbf{D}_{p p}(\mathbf{x}, \mathbf{n}(\mathbf{x}))$ and of $\mathbf{D}_{p u}(\mathbf{x}, \mathbf{n}(\mathbf{x}))$ :

$$
\mathbf{D}(\mathbf{x})=\mathbf{I}+\mathbf{D}_{p p}(\mathbf{x}, \mathbf{n}(\mathbf{x}))-\mathbf{D}_{p u}(\mathbf{x}, \mathbf{n}(\mathbf{x}))
$$

The free term coefficient $\mathbf{D}(\mathbf{x})$ is proved to depend on the material properties of the body, on the direction $\mathbf{n}(\mathbf{x})$ and on the angle $\varphi$ at the corner point. Furthermore, it will be proved that the sum of all unbounded logarithmic terms cancels out in the limit process, so that equation (2) is well defined even at a corner. Such an integral equation is however due to a property of the global elastic integral and not of each singular operator. Only in this view equation (2) still makes sense at a corner.

In order to prove all the statements above, the linear elastic isotropic constitutive law must be complied by all unknown fields. For all fields that do not fulfill

the constitutive law, all aforesaid properties do not seem to hold and equation (2) does not seem to make sense at a corner.

\section{Free terms arising from the hypersingular ker- nel}

This section aims at analyzing the limits 


$$
\begin{aligned}
& \lim _{\mathbf{x} \rightarrow \mathbf{x}_{0}}\left\{\int_{\Gamma_{\varepsilon}^{-}} \mathbf{G}_{p p}(\mathbf{r} ; \mathbf{n}(\mathbf{x}), \mathbf{l}(\mathbf{y})) \mathrm{d} \Gamma_{\mathbf{y}} \mathbf{u}\left(\mathbf{x}_{0}\right)\right. \\
& \left.+\int_{\Gamma_{\varepsilon}^{+}} \mathbf{G}_{p p}(\mathbf{r} ; \mathbf{n}(\mathbf{x}), \mathbf{l}(\mathbf{y})) \mathrm{d} \Gamma_{\mathbf{y}}\right\} \mathbf{u}\left(\mathbf{x}_{0}\right) \\
& \lim _{\mathbf{x} \rightarrow \mathbf{x}_{0}}\left\{\int_{\Gamma_{\varepsilon}^{-}} \mathbf{G}_{p p}(\mathbf{r} ; \mathbf{n}(\mathbf{x}), \mathbf{l}(\mathbf{y})) \boldsymbol{\epsilon}\left(\mathbf{x}_{0}\right) \mathbf{y} \mathrm{d} \Gamma_{\mathbf{y}}\right. \\
& \left.+\int_{\Gamma_{\varepsilon}^{+}} \mathbf{G}_{p p}(\mathbf{r} ; \mathbf{n}(\mathbf{x}), \mathbf{l}(\mathbf{y})) \boldsymbol{\epsilon}\left(\mathbf{x}_{0}\right) \mathbf{y} \mathrm{d} \Gamma_{\mathbf{y}}\right\}
\end{aligned}
$$

when $\mathbf{x} \in \Omega$. In the companion paper [1], the support of integrals (10)-(11) has been changed to $I_{\varepsilon}^{ \pm}$, in this way introducing approximations of different order $(O(\varepsilon)$ or $O(1))$ depending on the smoothness of $\Gamma_{\varepsilon}^{ \pm}$. This idea can be easily adapted to the case of corners, thus having almost $C^{n}$ boundaries, in the sense $y_{2}^{ \pm}\left(y_{1}\right) \in C^{n}\left(I_{\varepsilon}^{ \pm}\right), n=1,2$. As a consequence, analysis in sections 2.2 and 2.3 of reference [1] easily extends to corners. Accordingly, such results will not be repeated here, and reference is made to a corner formed by two straight lines.

Proposition 2.1 For solving integral integrals (10), (11) it is sufficient to solve the following:

$$
\int_{x_{1}-\frac{\varepsilon}{2}}^{x_{1}+\frac{\varepsilon}{2}} \mathbf{G}_{p p}(\mathbf{r} ; \mathbf{n}(\mathbf{x}) ; \mathbf{l}(\mathbf{y})) r_{1}^{i} \mathrm{~d} r_{1} \quad i=0,1 \quad \mathbf{x} \in \Omega
$$

Proof: $\quad$ With regard to $\Gamma_{\varepsilon}^{+}$and denoting with $\mathbf{e}_{1}$ the unit vector associated to the $y_{1}$ axis, in $\mathcal{L}^{+}$one has $\boldsymbol{\epsilon}\left(\mathbf{x}_{0}\right) \mathbf{y}=y_{1} \boldsymbol{\epsilon}\left(\mathbf{x}_{0}\right) \mathbf{e}_{1}+O\left(y_{1}^{2}\right)$. To evaluate integrals (10), (11) one therefore deals with the following integrals:

$$
\int_{0}^{\varepsilon} \mathbf{G}_{p p}(\mathbf{x}-\mathbf{z} ; \mathbf{n}(\mathbf{x}) ; \mathbf{l}(\mathbf{z})) y_{1}^{i} \mathrm{~d} y_{1} \quad i=0,1 \quad \mathbf{x} \in \Omega
$$

that can be performed by means of the variable change $\mathbf{r}=\mathbf{x}-\mathbf{z}$. The thesis follows after a reference change. Similar considerations lead to prove the thesis on $\Gamma_{\varepsilon}^{-}$.

Corners increase the amount of mathematical passages required by (10)-(11) with respect to a smooth boundary. Nevertheless, there are basically no new concepts in analytical integrations and details are collected in Appendix 1. New concepts come out in the limit process $\Omega \ni \mathbf{x} \rightarrow \mathbf{x}_{0} \in \Gamma_{\varepsilon}$. Defining with

$$
\mathbf{A}=\left(\begin{array}{cc}
\cos \varphi & -\sin \varphi \\
\sin \varphi & \cos \varphi
\end{array}\right)
$$

the matrix that governs the reference change between $\mathcal{L}^{-}$and $\mathcal{L}^{+}$, the following propositions hold (proofs in appendix 1): 
Proposition 2.2 For any $\varepsilon \in \mathbf{R}$ :

$$
\lim _{\mathbf{x} \rightarrow \mathbf{x}_{0}}\left\{\int_{-\varepsilon}^{0} \mathbf{G}_{p p}^{-} \mathrm{d} y_{1}+\mathbf{A}^{\top} \int_{0}^{\varepsilon} \mathbf{G}_{p p}^{+} \mathrm{d} y_{1} \mathbf{A}\right\}=f_{-\varepsilon}^{0} \mathbf{G}_{p p}^{-} \mathrm{d} y_{1}+\mathbf{A}^{\top} f_{0}^{\varepsilon} \mathbf{G}_{p p}^{+} \mathrm{d} y_{1} \mathbf{A}
$$

Proposition 2.3 For any $\varepsilon \in \mathbf{R}$ :

$$
\begin{gathered}
\lim _{\mathbf{x} \rightarrow \mathbf{x}_{0}}\left\{\int_{x_{1}^{-}-\frac{\varepsilon}{2}}^{x_{1}^{-}+\frac{\varepsilon}{2}} \mathbf{G}_{p p}^{-} \mathrm{d} r_{1}\left(x_{1}^{-}-\frac{\varepsilon}{2}\right) \boldsymbol{\epsilon}^{-}\left(\mathbf{x}_{0}\right) \mathbf{e}_{1}^{-}+\right. \\
\left.\left.\mathbf{A}^{\top} \int_{x_{1}^{+}-\frac{\varepsilon}{2}}^{x_{1}^{+}+\frac{\varepsilon}{2}} \mathbf{G}_{p p}^{+} \mathrm{d} r_{1}\left(x_{1}^{+}+\frac{\varepsilon}{2}\right) \mathbf{A} \boldsymbol{\epsilon}^{-}\left(\mathbf{x}_{0}\right) \mathbf{A}^{\top} \mathbf{e}_{1}^{+}\right\}=\mathbf{f} \mathbf{t}_{p p}, 3\right) \\
\lim _{\mathbf{x} \rightarrow \mathbf{x}_{0}}\left\{\int_{x_{1}^{-}-\frac{\varepsilon}{2}}^{x_{1}^{-}+\frac{\varepsilon}{2}} \mathbf{G}_{p p}^{-} r_{1} \mathrm{~d} r_{1} \boldsymbol{\epsilon}^{-}\left(\mathbf{x}_{0}\right) \mathbf{e}_{1}^{-}+\mathbf{A}^{\top} \int_{x_{1}^{+}-\frac{\varepsilon}{2}}^{x_{1}^{+}+\frac{\varepsilon}{2}} \mathbf{G}_{p p}^{+} r_{1} \mathrm{~d} r_{1} \mathbf{A} \boldsymbol{\epsilon}^{-}\left(\mathbf{x}_{0}\right) \mathbf{A}^{\top} \mathbf{e}_{1}^{+}\right\}= \\
f_{x_{1}^{-}-\frac{\varepsilon}{2}}^{x_{1}^{-}+\frac{\varepsilon}{2}} \mathbf{G}_{p p}^{-} r_{1} \mathrm{~d} r_{1} \boldsymbol{\epsilon}^{-}\left(\mathbf{x}_{0}\right) \mathbf{e}_{1}^{-}+\mathbf{A}^{\top} f_{x_{1}^{+}-\frac{\varepsilon}{2}}^{x_{1}^{+}+\frac{\varepsilon}{2}} \mathbf{G}_{p p}^{+} r_{1} \mathrm{~d} r_{1} \mathbf{A} \boldsymbol{\epsilon}^{-}\left(\mathbf{x}_{0}\right) \mathbf{A}^{\top} \mathbf{e}_{1}^{+}+ \\
\mathbf{f t}_{p p}^{1}+\mathbf{l}_{p p}^{1}(\varphi, \mathbf{n}, \boldsymbol{\epsilon}, G, \nu) \lim _{\mathbf{x} \rightarrow \mathbf{x}_{0}} \log \left\|\mathbf{x}-\mathbf{x}_{0}\right\|
\end{gathered}
$$

with vectors $\mathbf{f t}_{p p}^{0}, \mathbf{f t}_{p p}^{1}$, and $\mathbf{l}_{p p}^{1}$ defined by comparison with equations (27), (30) in Appendix 1. Differently from the smooth case, the limit to the boundary (14) shows an unbounded logarithmic term. It is questionable therefore if equation (2), formulated in terms of HFP and CPV, still makes sense in the presence of corners. Indeed, as proved later on in the paper, the logarithmic unbounded term cancels out with an unbounded counterpart arising from the strongly singular kernel. This event may give a sense to the equation (2) on merely continuous boundaries, owing to a property of the global (elastic) problem. The finite part formulation should therefore be intended for corners as an extension of equation (2) which may be proved rigorously only for smooth boundaries, because identity (16) in paper [1] cannot be extended in the presence of corners.

Vectors $\mathbf{f t}_{p p}^{0}, \mathbf{f t}_{p p}^{1}$ are independent on $\varepsilon$ and therefore act as a free term. Unfortunately they depend on the direction by which $\mathbf{x}$ approaches $\mathbf{x}_{0}$ and are not consistent with the definition of a limit process (see also figure 2). Such a dependency is proved to vanish (see Section 4) with a counterpart arising from the strongly singular kernel. Only in this way of thinking one may conclude that the term $-\mathbf{f t}_{p p}^{0}-\mathbf{f t}_{p p}^{1}$ is the extension of the hypersingular free term contribution (13) in paper [1] in the presence of corners.

\section{Free terms arising from the strongly singular kernel.}

Consider the integral $(\mathrm{x} \in \Omega)$

$$
\lim _{\mathbf{x} \rightarrow \mathbf{x}_{0}}\left\{\int_{\Gamma_{\varepsilon}^{-}} \mathbf{G}_{p u}(\mathbf{r} ; \mathbf{n}(\mathbf{x})) \mathrm{d} \Gamma \mathbf{p}^{-}\left(\mathbf{x}_{0}\right)+\int_{\Gamma_{\varepsilon}^{+}} \mathbf{G}_{p u}(\mathbf{r} ; \mathbf{n}(\mathbf{x})) \mathrm{d} \Gamma \mathbf{p}^{+}\left(\mathbf{x}_{0}\right)\right\}
$$



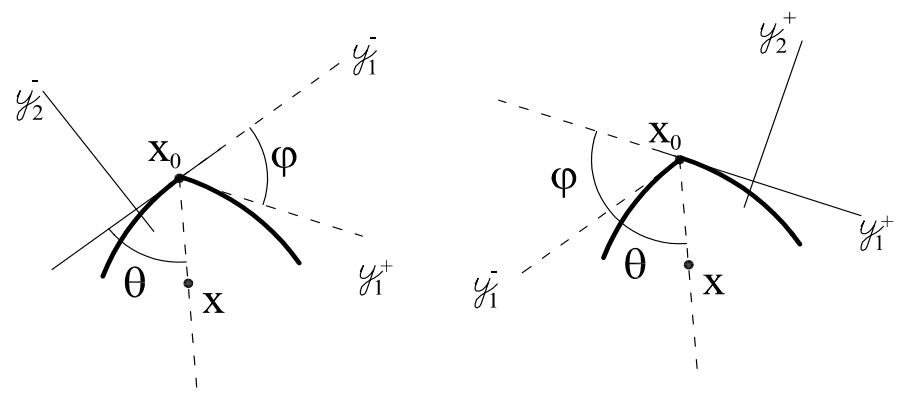

Figure 2: Limit process $\Omega \ni \mathbf{x} \rightarrow \mathbf{x}_{0} \in \Gamma$.

As stated in the introduction, $\mathbf{p}^{-}\left(\mathbf{x}_{0}\right)$ stands for $\mathbf{p}^{-}\left(\mathbf{x}_{0}, \mathbf{l}^{-}\left(\mathbf{x}_{0}\right)\right)$; since at $\mathbf{x}_{0}$ normals to the boundary are not defined, $\mathbf{l}^{-}\left(\mathbf{x}_{0}\right)$ is here intended in a limit sense, i.e.

$$
\mathbf{l}^{-}\left(\mathbf{x}_{0}\right) \stackrel{\text { def }}{=} \lim _{\mathbf{y} \rightarrow \mathbf{x}_{0}} \mathbf{l}(\mathbf{y}) \quad \mathbf{y} \in \Gamma_{\varepsilon}^{-}
$$

and the same holds for $\mathbf{l}^{+}\left(\mathbf{x}_{0}\right)$.

Proposition 3.1 If

$$
\sqrt{1+y_{2}^{\prime}\left(y_{1}\right)^{2}}=1+O\left(y_{1}\right) \quad \forall y_{1} \in[-\varepsilon, 0[\cup] 0, \varepsilon]
$$

then for solving integral (15) it is sufficient to solve integrals:

$$
\int_{x_{1}-\frac{\varepsilon}{2}}^{x_{1}+\frac{\varepsilon}{2}} \mathbf{G}_{p u}(\mathbf{r} ; \mathbf{n}(\mathbf{x})) \mathrm{d} r_{1} \quad \mathbf{x} \in \Omega
$$

Proof: $\quad$ Denoting with $r=\|\mathbf{x}-\mathbf{y}\|$ and taking $\mathbf{x} \notin \Gamma_{\varepsilon}^{+}$, one writes in $\mathcal{L}^{+}$:

$$
\begin{aligned}
\int_{\Gamma_{\varepsilon}^{+}} \frac{f(\mathbf{y})}{r} \mathrm{~d} \Gamma \stackrel{\text { def }}{=} \int_{s_{0}}^{l} \frac{f(\tilde{\mathbf{y}}(s))}{r} \mathrm{~d} s & =\int_{0}^{\varepsilon} \frac{f\left(\mathbf{y}\left(y_{1}\right)\right)}{r} \sqrt{1+y_{2}^{\prime}\left(y_{1}\right)^{2}} \mathrm{~d} y_{1} \\
& =\int_{0}^{\varepsilon} \frac{f\left(\mathbf{y}\left(y_{1}\right)\right)}{r} \mathrm{~d} y_{1}+O(\varepsilon)
\end{aligned}
$$

for any $f\left(\mathbf{y}\left(y_{1}\right)\right) \in C^{0}\left(I_{\varepsilon}^{+}\right)$in view of the adopted hypothesis.

The strongly singular kernel shows the same peculiarities (see proposition 2.3) of the hypersingular kernel with regard to the free term analysis, owing to the following result, which is proved in appendix 2: 
Proposition 3.2 For any $\varepsilon \in \mathbf{R}$ :

$$
\begin{gathered}
\lim _{\mathbf{x} \rightarrow \mathbf{x}_{0}}\left\{\int_{-\varepsilon}^{0} \mathbf{G}_{p u}^{-} \mathrm{d} y_{1} \boldsymbol{\sigma}^{-}\left(\mathbf{x}_{0}\right) \mathbf{e}_{2}^{-}+\mathbf{A}^{\top} \int_{0}^{\varepsilon} \mathbf{G}_{p u}^{+} \mathrm{d} y_{1} \mathbf{A} \boldsymbol{\sigma}^{-}\left(\mathbf{x}_{0}\right) \mathbf{A}^{\top} \mathbf{l}^{+}\right\}= \\
f_{-\varepsilon}^{0} \mathbf{G}_{p u}^{-} \mathrm{d} y_{1} \boldsymbol{\sigma}^{-}\left(\mathbf{x}_{0}\right) \mathbf{e}_{2}^{-}+\mathbf{A}^{\top} f_{0}^{\varepsilon} \mathbf{G}_{p u}^{+} \mathrm{d} y_{1} \mathbf{A} \boldsymbol{\sigma}^{-}\left(\mathbf{x}_{0}\right) \mathbf{A}^{\top} \mathbf{l}^{+}+ \\
\mathbf{f t}_{p u}-\mathbf{l}_{p u}^{1}(\varphi, \mathbf{n}, \boldsymbol{\sigma}, G, \nu) \lim _{\mathbf{x} \rightarrow \mathbf{x}_{0}} \log \left\|\mathbf{x}-\mathbf{x}_{0}\right\|
\end{gathered}
$$

The vector $\mathbf{f t}_{p u}$ is defined by comparison with eq. (32) in appendix 2 . The limit (17) is an essential result for the free term analysis: it shows that, in the presence of corners, the strongly singular integral produces unbounded terms that do not cancel out in the limit process $\Omega \ni \mathbf{x} \rightarrow \mathbf{x}_{0} \in \Gamma$.

The limit (17) gives Cauchy's principal value, plus an unbounded logarithmic term and the vector $\mathbf{f t}_{p u}$, which plays the role of a free term being independent on $\varepsilon$. Similarly to $\mathbf{f t}_{p p}$, the vector $\mathbf{f t}_{p u}$ depends on the selected direction in the limit process $\Omega \ni \mathbf{x} \rightarrow \mathbf{x}_{0} \in \Gamma$, therefore it cannot be defined as a result of a limit process for it is not consistent with a definition of limit (see also figure 2).

\section{Properties of free terms coefficients}

In this section, some properties of the free terms are stated, under the hypothesis of validity of the linear isotropic elastic constitutive law:

$$
\boldsymbol{\epsilon}=-\frac{\nu}{E} \operatorname{tr}(\boldsymbol{\sigma}) \mathbf{I}+\frac{1+\nu}{E} \boldsymbol{\sigma}
$$

The goal is to extend Proposition 4.1 in [1] to the case of corners.

Proposition 4.1 If displacement and stress fields satisfy the constitutive equation (18), then:

$$
\mathbf{l}_{p p}^{1}(\varphi, \mathbf{n}, \boldsymbol{\epsilon}(\boldsymbol{\sigma}), G, \nu)=\mathbf{l}_{p u}^{1}(\varphi, \mathbf{n}, \boldsymbol{\sigma}, G, \nu)
$$

Proof: $\quad$ See appendices 1, 2.

As a main consequence of Proposition 4.1, the logarithmic unbounded term of the hypersingular integral cancel out with an unbounded counterpart arising from the strongly singular kernel. This event may give sense to the equation (2) on merely continuous boundaries, owing to a property of the global (elastic) problem. Though, Proposition 4.1 applies only to displacement and stress fields that satisfy the constitutive equation (18). Therefore it holds for the exact solution fields $\mathbf{u}(\mathbf{y})$, $\mathbf{p}(\mathbf{y})$ but generally not for the approximated fields that pertain to the numerical solution. Accordingly, an unbounded behavior at corners for the post-processing stress evaluation at a corner seems to be unavoidable. 
Proposition 4.2 If displacement and stress fields satisfy the constitutive equation (18), then the sum

$$
\mathbf{f t}_{p u}+\mathbf{f t}_{p p}^{0}+\mathbf{f t}_{p p}^{1}
$$

does not depend on the direction $\theta$ (see figure 2).

Proof: The thesis is proved by direct substitution, making use of the constitutive law (18) and of the definitions in Appendices 1, 2:

$$
\mathbf{f t}_{p u}=\mathbf{a}_{p u}+\mathbf{s}_{p u}, \quad \mathbf{f t}_{p p}^{0}=-\mathbf{a}_{p p}^{0}-\mathbf{s}_{p p}^{0}, \quad \mathbf{f t}_{p p}^{1}=-\mathbf{a}_{p p}^{1}-\mathbf{s}_{p p}^{1}-\mathbf{h}_{p p}^{1}
$$

It comes out:

$$
\begin{aligned}
\mathbf{f t}_{p u} & +\mathbf{f t}_{p p}^{0}+\mathbf{f t}_{p p}^{1}=\frac{1}{16 \pi(-1+\nu)}\{ \\
& 8(\varphi+\pi)\left(n_{1} \sigma_{11}+n_{2} \sigma_{21}\right)(-1+\nu)+6 \sigma_{21} n_{1}+ \\
& n_{2}\left(3 \sigma_{22}+\sigma_{11}(5-6 \nu)-6 \sigma_{22} \nu\right)+ \\
& +\left(-4 n_{1} \sigma_{21}+2 n_{2}\left(\sigma_{11}+\sigma_{22}\right)(-2+3 \nu)\right) \cos (2 \varphi)+ \\
& +2 n_{1}\left(-3 \sigma_{11}-\sigma_{22}+3\left(\sigma_{11}+\sigma_{22}\right) \nu\right) \sin (2 \varphi)+ \\
& +\left(-2 n_{1} \sigma_{21}+n_{2}\left(-\sigma_{11}+\sigma_{22}\right)\right) \cos (4 \varphi)+\left(2 n_{2} \sigma_{21}+n_{1}\left(-\sigma_{11}+\sigma_{22}\right)\right) \sin (4 \varphi) \\
& 8(\varphi+\pi)\left(n_{1} \sigma_{21}+n_{2} \sigma_{22}\right)(-1+\nu)+2 n_{2} \sigma_{21}+ \\
& n_{1}\left(5 \sigma_{11}+3 \sigma_{22}-6\left(\sigma_{11}+\sigma_{22}\right) \nu\right)+ \\
& +\left(-4 n_{2} \sigma_{21}+2 n_{1}\left(\sigma_{11}+\sigma_{22}\right)(-2+3 \nu)\right) \cos (2 \varphi)+ \\
& -2 n_{2}\left(-\sigma_{11}-3 \sigma_{22}+3\left(\sigma_{11}+\sigma_{22}\right) \nu\right) \sin (2 \varphi)+ \\
& \left.+\left(2 n_{2} \sigma_{21}+n_{1}\left(-\sigma_{11}+\sigma_{22}\right)\right) \cos (4 \varphi)+\left(2 n_{1} \sigma_{21}+n_{2}\left(\sigma_{11}-\sigma_{22}\right)\right) \sin (4 \varphi)\right\}
\end{aligned}
$$

Proposition 4.2 implies that all the limit processes behind the terms $\mathbf{f t}_{p u}, \mathbf{f t}_{p p}^{0}$, and $\mathrm{ft}_{p p}^{1}$ make sense when their sum is considered, and the sum $\mathbf{f t}_{p u}+\mathbf{f t}_{p p}^{0}+\mathbf{f t}_{p p}^{1}$ is the result of such a limit process. The next statement extends Proposition 4.1 in [1] to the case of corners:

Proposition 4.3 Let $\mathbf{p}\left(\mathbf{x}_{0}\right)$ refer to the direction $\mathbf{n}\left(\mathbf{x}_{0}\right)$ and displacement and stress fields satisfy the constitutive equation (18). Then a matrix $\mathbf{D}$ exists such that equation (2) holds at $\mathbf{x}_{0} \in \Gamma_{\varepsilon}$.

Proof: $\quad$ Take the local orthogonal systems $\mathcal{L}^{+}, \mathcal{L}^{-}$as usual, see figure 1-b, and for simplicity assume $\mathcal{L}^{-}$to be the absolute coordinate system. Consider $\mathbf{x} \in \Omega$ and the $H I$ (1):

$$
\begin{aligned}
& \mathbf{p}(\mathbf{x}, \mathbf{n}(\mathbf{x}))=\int_{\Gamma} \mathbf{G}_{p u}(\mathbf{r} ; \mathbf{n}(\mathbf{x})) \mathbf{p}(\mathbf{y}) \mathrm{d} \Gamma_{\mathbf{y}}-\int_{\Gamma} \mathbf{G}_{p p}(\mathbf{r} ; \mathbf{n}(\mathbf{x}) ; \mathbf{l}(\mathbf{y})) \mathbf{u}(\mathbf{y}) \mathrm{d} \Gamma_{\mathbf{y}} \\
& \quad=\int_{\Gamma \backslash \Gamma_{\varepsilon}} \mathbf{G}_{p u}(\mathbf{r} ; \mathbf{n}(\mathbf{x})) \mathbf{p}(\mathbf{y}) \mathrm{d} \Gamma_{\mathbf{y}}+\int_{\Gamma_{\varepsilon}^{-}} \mathbf{G}_{p u}()\left[\mathbf{p}(\mathbf{y})-\mathbf{p}^{-}\left(\mathbf{x}_{0}\right)\right] \mathrm{d} \Gamma \\
& \quad+\int_{\Gamma_{\varepsilon}^{-}} \mathbf{G}_{p u}() \mathrm{d} \Gamma \mathbf{p}^{-}\left(\mathbf{x}_{0}\right)+\int_{\Gamma_{\varepsilon}^{+}} \mathbf{G}_{p u}()\left[\mathbf{p}(\mathbf{y})-\mathbf{p}^{+}\left(\mathbf{x}_{0}\right)\right] \mathrm{d} \Gamma
\end{aligned}
$$




$$
\begin{aligned}
& +\int_{\Gamma_{\varepsilon}^{+}} \mathbf{G}_{p u}() \mathrm{d} \Gamma \mathbf{p}^{+}\left(\mathbf{x}_{0}\right)-\int_{\Gamma \backslash \Gamma_{\varepsilon}} \mathbf{G}_{p p}(\mathbf{r} ; \mathbf{n}(\mathbf{x}) ; \mathbf{l}(\mathbf{y})) \mathbf{u}(\mathbf{y}) \mathrm{d} \Gamma_{\mathbf{y}} \\
& -\int_{\Gamma_{\varepsilon}} \mathbf{G}_{p p}()\left[\mathbf{u}(\mathbf{y})-\mathbf{u}\left(\mathbf{x}_{0}\right)-\boldsymbol{\epsilon}\left(\mathbf{x}_{0}\right)\left(\mathbf{y}-\mathbf{x}_{0}\right)\right] \mathrm{d} \Gamma \\
& -\int_{\Gamma_{\varepsilon}} \mathbf{G}_{p p}() \mathrm{d} \Gamma \mathbf{u}\left(\mathbf{x}_{0}\right)-\int_{\Gamma_{\varepsilon}} \mathbf{G}_{p p}() \boldsymbol{\epsilon}\left(\mathbf{x}_{0}\right) \mathbf{y} \mathrm{d} \Gamma
\end{aligned}
$$

$\mathbf{p}^{-}\left(\mathbf{x}_{0}\right)$ stands for $\mathbf{p}^{-}\left(\mathbf{x}_{0}, \mathbf{l}^{-}\left(\mathbf{x}_{0}\right)\right)$; since at $\mathbf{x}_{0}$ normals to the boundary are not defined, $\mathbf{l}^{-}\left(\mathbf{x}_{0}\right)$ is here intended in a limit sense, i.e.

$$
\mathbf{l}^{-}\left(\mathbf{x}_{0}\right) \stackrel{\text { def }}{=} \lim _{\mathbf{y} \rightarrow \mathbf{x}_{0}} \mathbf{l}(\mathbf{y}) \quad \mathbf{y} \in \Gamma_{\varepsilon}^{-}
$$

and the same holds for $\mathbf{1}^{+}\left(\mathbf{x}_{0}\right)$. Take the limit to the boundary $\Omega \ni \mathbf{x} \rightarrow \mathbf{x}_{0}$ :

$$
\begin{aligned}
& \mathbf{p}\left(\mathbf{x}_{0}, \mathbf{n}\left(\mathbf{x}_{0}\right)\right)= \\
& \int_{\Gamma \backslash \Gamma_{\varepsilon}} \mathbf{G}_{p u}\left(\mathbf{x}_{0}-\mathbf{y} ; \mathbf{n}\left(\mathbf{x}_{0}\right)\right) \mathbf{p}(\mathbf{y}) \mathrm{d} \Gamma_{\mathbf{y}}-\int_{\Gamma \backslash \Gamma_{\varepsilon}} \mathbf{G}_{p p}\left(\mathbf{x}_{0}-\mathbf{y} ; \mathbf{n}\left(\mathbf{x}_{0}\right) ; \mathbf{l}(\mathbf{y})\right) \mathbf{u}(\mathbf{y}) \mathrm{d} \Gamma_{\mathbf{y}} \\
& +\lim _{\mathbf{x} \rightarrow \mathbf{x}_{0}}\left\{\int_{\Gamma_{\varepsilon}^{-}} \mathbf{G}_{p u}(\mathbf{x}-\mathbf{y} ; \mathbf{n}(\mathbf{x}))\left[\mathbf{p}(\mathbf{y})-\mathbf{p}^{-}\left(\mathbf{x}_{0}\right)\right] \mathrm{d} \Gamma\right. \\
& \left.+\int_{\Gamma_{\varepsilon}^{+}} \mathbf{G}_{p u}(\mathbf{x}-\mathbf{y} ; \mathbf{n}(\mathbf{x}))\left[\mathbf{p}(\mathbf{y})-\mathbf{p}^{+}\left(\mathbf{x}_{0}\right)\right] \mathrm{d} \Gamma\right\} \\
& +\lim _{\mathbf{x} \rightarrow \mathbf{x}_{0}}\left\{\int_{\Gamma_{\varepsilon}^{-}} \mathbf{G}_{p u}(\mathbf{x}-\mathbf{y} ; \mathbf{n}(\mathbf{x})) \mathrm{d} \Gamma\right\} \mathbf{p}^{-}\left(\mathbf{x}_{0}\right) \\
& +\lim _{\mathbf{x} \rightarrow \mathbf{x}_{0}}\left\{\int_{\Gamma_{\varepsilon}^{+}} \mathbf{G}_{p u}(\mathbf{x}-\mathbf{y} ; \mathbf{n}(\mathbf{x})) \mathrm{d} \Gamma\right\} \mathbf{p}^{+}\left(\mathbf{x}_{0}\right) \\
& -\lim _{\mathbf{x} \rightarrow \mathbf{x}_{0}}\left\{\int_{\Gamma_{\varepsilon}} \mathbf{G}_{p p}(\mathbf{r} ; \mathbf{n}(\mathbf{x}) ; \mathbf{l}(\mathbf{y}))\left[\mathbf{u}(\mathbf{y})-\mathbf{u}\left(\mathbf{x}_{0}\right)-\boldsymbol{\epsilon}\left(\mathbf{x}_{0}\right)\left(\mathbf{y}-\mathbf{x}_{0}\right)\right] \mathrm{d} \Gamma\right\} \\
& -\lim _{\mathbf{x} \rightarrow \mathbf{x}_{0}}\left\{\int_{\Gamma_{\varepsilon}} \mathbf{G}_{p p}(\mathbf{r} ; \mathbf{n}(\mathbf{x}) ; \mathbf{l}(\mathbf{y})) \mathrm{d} \Gamma\right\} \mathbf{u}\left(\mathbf{x}_{0}\right) \\
& -\lim _{\mathbf{x} \rightarrow \mathbf{x}_{0}}\left\{\int_{\Gamma_{\varepsilon}} \mathbf{G}_{p p}(\mathbf{r} ; \mathbf{n}(\mathbf{x}) ; \mathbf{l}(\mathbf{y})) \boldsymbol{\epsilon}\left(\mathbf{x}_{0}\right) \mathbf{y} \mathrm{d} \Gamma\right\}
\end{aligned}
$$

Denote with

$$
\begin{aligned}
& f_{\Gamma_{\varepsilon}^{-}} \mathbf{G}_{p u}\left(\mathbf{x}_{0}-\mathbf{y} ; \mathbf{n}\left(\mathbf{x}_{0}\right)\right) \mathrm{d} \Gamma \stackrel{\text { def }}{=} f_{-\varepsilon}^{0} \mathbf{G}_{p u}^{-}\left(\mathbf{x}_{0}-\mathbf{y} ; \mathbf{n}\left(\mathbf{x}_{0}\right)\right) \mathrm{d} y_{1} \\
& f_{\Gamma_{\varepsilon}^{+}} \mathbf{G}_{p u}\left(\mathbf{x}_{0}-\mathbf{y} ; \mathbf{n}\left(\mathbf{x}_{0}\right)\right) \mathrm{d} \Gamma \stackrel{\text { def }}{=} \mathbf{A}^{\top} f_{0}^{\varepsilon} \mathbf{G}_{p u}^{+}\left(\mathbf{x}_{0}-\mathbf{y} ; \mathbf{n}\left(\mathbf{x}_{0}\right)\right) \mathrm{d} y_{1} \mathbf{A} \\
& f_{\Gamma_{\varepsilon}} \mathbf{G}_{p p}\left(\mathbf{x}_{0}-\mathbf{y} ; \mathbf{n}(\mathbf{x}) ; \mathbf{l}(\mathbf{y})\right) \mathrm{d} \Gamma \stackrel{\text { def }}{=} f_{-\varepsilon}^{0} \mathbf{G}_{p p}^{-}\left(\mathbf{x}_{0}-\mathbf{y} ; \mathbf{n}(\mathbf{x}) ; \mathbf{l}(\mathbf{y})\right) \mathrm{d} y_{1}+ \\
& \mathbf{A}^{\top} f_{0}^{\varepsilon} \mathbf{G}_{p p}^{+}\left(\mathbf{x}_{0}-\mathbf{y} ; \mathbf{n}(\mathbf{x}) ; \mathbf{l}(\mathbf{y})\right) \mathrm{d} y_{1} \mathbf{A}
\end{aligned}
$$




$$
\begin{aligned}
& =\int_{x_{1}^{-}-\frac{\varepsilon}{2}}^{x_{1}^{-}+\frac{\varepsilon}{2}} \mathbf{G}_{p p}^{-}\left(\mathbf{x}_{0}-\mathbf{y} ; \mathbf{n}(\mathbf{x}) ; \mathbf{l}(\mathbf{y})\right) \mathrm{d} r_{1}^{-}+\mathbf{A}^{\top} \int_{x_{1}^{+}-\frac{\varepsilon}{2}}^{x_{1}^{+}+\frac{\varepsilon}{2}} \mathbf{G}_{p p}^{+}\left(\mathbf{x}_{0}-\mathbf{y} ; \mathbf{n}(\mathbf{x}) ; \mathbf{l}(\mathbf{y})\right) \mathrm{d} r_{1}^{+} \mathbf{A} \\
& f_{\Gamma_{\varepsilon}} \mathbf{G}_{p p}\left(\mathbf{x}_{0}-\mathbf{y} ; \mathbf{n}(\mathbf{x}) ; \mathbf{l}(\mathbf{y})\right) \boldsymbol{\epsilon}\left(\mathbf{x}_{0}\right) \mathbf{y} \mathrm{d} \Gamma \stackrel{d e f}{=} \\
& \stackrel{\text { def }}{=} f_{-\varepsilon}^{0} \mathbf{G}_{p p}^{-}\left(\mathbf{x}_{0}-\mathbf{y} ; \mathbf{n}(\mathbf{x}) ; \mathbf{l}(\mathbf{y})\right) \boldsymbol{\epsilon}^{-}\left(\mathbf{x}_{0}\right) \mathbf{y}^{-} \mathrm{d} y_{1}+ \\
& \quad \mathbf{A}^{\top} f_{0}^{\varepsilon} \mathbf{G}_{p p}^{+}\left(\mathbf{x}_{0}-\mathbf{y} ; \mathbf{n}(\mathbf{x}) ; \mathbf{l}(\mathbf{y})\right) \boldsymbol{\epsilon}^{+}\left(\mathbf{x}_{0}\right) \mathbf{y}^{+} \mathrm{d} y_{1} \\
& =f_{-\varepsilon}^{0} \mathbf{G}_{p p}^{-}\left(\mathbf{x}_{0}-\mathbf{y} ; \mathbf{n}(\mathbf{x}) ; \mathbf{l}(\mathbf{y})\right) y_{1}^{-} \mathrm{d} y_{1} \boldsymbol{\epsilon}^{-}\left(\mathbf{x}_{0}\right) \mathbf{e}_{1}^{-}+ \\
& \quad \mathbf{A}^{\top} f_{0}^{\varepsilon} \mathbf{G}_{p p}^{+}\left(\mathbf{x}_{0}-\mathbf{y} ; \mathbf{n}(\mathbf{x}) ; \mathbf{l}(\mathbf{y})\right) y_{1}^{+} \mathrm{d} y_{1} \boldsymbol{\epsilon}^{+}\left(\mathbf{x}_{0}\right) \mathbf{e}_{1}^{+} \\
& =-f_{x_{1}^{-}-\frac{\varepsilon}{2}}^{x_{1}^{-}+\frac{\varepsilon}{2}} \mathbf{G}_{p p}^{-}() r_{1}^{-} \mathrm{d} r_{1} \boldsymbol{\epsilon}^{-}\left(\mathbf{x}_{0}\right) \mathbf{e}_{1}^{-}-\mathbf{A}^{\top} f_{x_{1}^{+}-\frac{\varepsilon}{2}}^{x_{1}^{+}+\frac{\varepsilon}{2}} \mathbf{G}_{p p}^{+}() r_{1}^{+} \mathrm{d} r_{1} \mathbf{A} \boldsymbol{\epsilon}^{-}\left(\mathbf{x}_{0}\right) \mathbf{A}^{\top} \mathbf{e}_{1}^{+}
\end{aligned}
$$

From equations (17), (12), two terms in the limit above simplify as follows:

$$
\begin{gathered}
\lim _{\mathbf{x} \rightarrow \mathbf{x}_{0}}\left\{\int_{\Gamma_{\varepsilon}^{-}} \mathbf{G}_{p u}(\mathbf{x}-\mathbf{y} ; \mathbf{n}(\mathbf{x})) \mathrm{d} \Gamma\right\} \mathbf{p}^{-}\left(\mathbf{x}_{0}\right)+\lim _{\mathbf{x} \rightarrow \mathbf{x}_{0}}\left\{\int_{\Gamma_{\varepsilon}^{+}} \mathbf{G}_{p u}(\mathbf{x}-\mathbf{y} ; \mathbf{n}(\mathbf{x})) \mathrm{d} \Gamma\right\} \mathbf{p}^{+}\left(\mathbf{x}_{0}\right)= \\
f_{\Gamma_{\varepsilon}^{-}} \mathbf{G}_{p u}\left(\mathbf{x}_{0}-\mathbf{y} ; \mathbf{n}\left(\mathbf{x}_{0}\right)\right) \mathrm{d} \Gamma \mathbf{p}^{-}\left(\mathbf{x}_{0}\right)+f_{\Gamma_{\varepsilon}^{+}} \mathbf{G}_{p u}\left(\mathbf{x}_{0}-\mathbf{y} ; \mathbf{n}\left(\mathbf{x}_{0}\right)\right) \mathrm{d} \Gamma \mathbf{p}^{+}\left(\mathbf{x}_{0}\right)+ \\
+\mathbf{f t}_{p u}-\mathbf{l}_{p p}^{1}(\varphi, \mathbf{n}, \boldsymbol{\epsilon}(\boldsymbol{\sigma}), G, \nu) \lim _{\mathbf{x} \rightarrow \mathbf{x}_{0}} \log \left\|\mathbf{x}-\mathbf{x}_{0}\right\| \\
\lim _{\mathbf{x} \rightarrow \mathbf{x}_{0}}\left\{\int_{\Gamma_{\varepsilon}} \mathbf{G}_{p p}(\mathbf{r} ; \mathbf{n}(\mathbf{x}) ; \mathbf{l}(\mathbf{y})) \mathrm{d} \Gamma\right\} \mathbf{u}\left(\mathbf{x}_{0}\right)=f_{\Gamma_{\varepsilon}} \mathbf{G}_{p p}\left(\mathbf{x}_{0}-\mathbf{y} ; \mathbf{n}(\mathbf{x}) ; \mathbf{l}(\mathbf{y})\right) \mathrm{d} \Gamma \mathbf{u}\left(\mathbf{x}_{0}\right)
\end{gathered}
$$

Furthermore, making use of equations (26), (14)

$$
\begin{aligned}
& \lim _{\mathbf{x} \rightarrow \mathbf{x}_{0}}\left\{\int_{\Gamma_{\varepsilon}} \mathbf{G}_{p p}(\mathbf{r} ; \mathbf{n}(\mathbf{x}) ; \mathbf{l}(\mathbf{y})) \boldsymbol{\epsilon}\left(\mathbf{x}_{0}\right) \mathbf{y} \mathrm{d} \Gamma\right\}= \\
& =\lim _{\mathbf{x} \rightarrow \mathbf{x}_{0}}\left\{\int_{-\varepsilon}^{0} \mathbf{G}_{p p}^{-}(\mathbf{r} ; \mathbf{n}(\mathbf{x}) ; \mathbf{l}(\mathbf{y})) y_{1}^{-} \mathrm{d} y_{1} \boldsymbol{\epsilon}^{-}\left(\mathbf{x}_{0}\right) \mathbf{e}_{1}^{-}+\right. \\
& \left.\mathbf{A}^{\top} \int_{0}^{\varepsilon} \mathbf{G}_{p p}^{+}(\mathbf{r} ; \mathbf{n}(\mathbf{x}) ; \mathbf{l}(\mathbf{y})) y_{1}^{+} \mathrm{d} y_{1} \boldsymbol{\epsilon}^{+}\left(\mathbf{x}_{0}\right) \mathbf{e}_{1}^{+}\right\} \\
& =\lim _{\mathbf{x} \rightarrow \mathbf{x}_{0}}\left\{\left(\int_{x_{1}^{-}-\frac{\varepsilon}{2}}^{x_{1}^{-}+\frac{\varepsilon}{2}} \mathbf{G}_{p p}^{-}(\mathbf{r} ; \mathbf{n}(\mathbf{x}) ; \mathbf{l}(\mathbf{y})) \mathrm{d} r_{1}^{-}\left(x_{1}^{-}-\frac{\varepsilon}{2}\right)\right.\right. \\
& \left.\quad-\int_{x_{1}^{-}-\frac{\varepsilon}{2}}^{x_{1}^{-}+\frac{\varepsilon}{2}} \mathbf{G}_{p p}^{-}(\mathbf{r} ; \mathbf{n}(\mathbf{x}) ; \mathbf{l}(\mathbf{y})) r_{1}^{-} \mathrm{d} r_{1}^{-}\right) \boldsymbol{\epsilon}^{-}\left(\mathbf{x}_{0}\right) \mathbf{e}_{1}^{-}+ \\
& \mathbf{A}^{\top}\left(\int_{x_{1}^{+}-\frac{\varepsilon}{2}}^{x_{1}^{+}+\frac{\varepsilon}{2}} \mathbf{G}_{p p}^{+}(\mathbf{r} ; \mathbf{n}(\mathbf{x}) ; \mathbf{l}(\mathbf{y})) \mathrm{d} r_{1}^{+}\left(x_{1}^{+}+\frac{\varepsilon}{2}\right)\right.
\end{aligned}
$$




$$
\begin{array}{r}
\left.-\int_{x_{1}^{+}-\frac{\varepsilon}{2}}^{x_{1}^{+}+\frac{\varepsilon}{2}} \mathbf{G}_{p p}^{+}(\mathbf{r} ; \mathbf{n}(\mathbf{x}) ; \mathbf{l}(\mathbf{y})) r_{1}^{+} \mathrm{d} r_{1}^{+}\right) \boldsymbol{\epsilon}^{+}\left(\mathbf{x}_{0}\right) \mathbf{e}_{1}^{+} \\
\stackrel{(26),(14)}{=}-\mathbf{f t}_{p p}^{0}+f_{\Gamma_{\varepsilon}} \mathbf{G}_{p p}\left(\mathbf{x}_{0}-\mathbf{y} ; \mathbf{n}(\mathbf{x}) ; \mathbf{l}(\mathbf{y})\right) \boldsymbol{\epsilon}\left(\mathbf{x}_{0}\right) \mathbf{y} \mathrm{d} \Gamma-\mathbf{f t}_{p p}^{1} \\
-\mathbf{l}_{p p}^{1}(\varphi, \mathbf{n}, \boldsymbol{\epsilon}, G, \nu) \lim _{\mathbf{x} \rightarrow \mathbf{x}_{0}} \log \left\|\mathbf{x}-\mathbf{x}_{0}\right\|
\end{array}
$$

By substituting all partial results above, it is straightforward to get:

$$
\begin{aligned}
& \mathbf{p}\left(\mathbf{x}_{0}, \mathbf{n}\left(\mathbf{x}_{0}\right)\right)= \\
& \quad \int_{\Gamma \backslash \Gamma_{\varepsilon}} \mathbf{G}_{p u}\left(\mathbf{x}_{0}-\mathbf{y} ; \mathbf{n}\left(\mathbf{x}_{0}\right)\right) \mathbf{p}(\mathbf{y}) \mathrm{d} \Gamma_{\mathbf{y}}-\int_{\Gamma \backslash \Gamma_{\varepsilon}} \mathbf{G}_{p p}\left(\mathbf{x}_{0}-\mathbf{y} ; \mathbf{n}\left(\mathbf{x}_{0}\right) ; \mathbf{l}(\mathbf{y})\right) \mathbf{u}(\mathbf{y}) \mathrm{d} \Gamma_{\mathbf{y}} \\
& +\lim _{\mathbf{x} \rightarrow \mathbf{x}_{0}}\left\{\int_{\Gamma_{\varepsilon}^{-}} \mathbf{G}_{p u}(\mathbf{x}-\mathbf{y} ; \mathbf{n}(\mathbf{x}))\left[\mathbf{p}(\mathbf{y})-\mathbf{p}^{-}\left(\mathbf{x}_{0}\right)\right] \mathrm{d} \Gamma\right. \\
& \left.\quad+\int_{\Gamma_{\varepsilon}^{+}} \mathbf{G}_{p u}(\mathbf{x}-\mathbf{y} ; \mathbf{n}(\mathbf{x}))\left[\mathbf{p}(\mathbf{y})-\mathbf{p}^{+}\left(\mathbf{x}_{0}\right)\right] \mathrm{d} \Gamma\right\} \\
& +f_{\Gamma_{\varepsilon}^{-}} \mathbf{G}_{p u}\left(\mathbf{x}_{0}-\mathbf{y} ; \mathbf{n}\left(\mathbf{x}_{0}\right)\right) \mathrm{d} \Gamma \mathbf{p}^{-}\left(\mathbf{x}_{0}\right)+f_{\Gamma_{\varepsilon}^{+}} \mathbf{G}_{p u}\left(\mathbf{x}_{0}-\mathbf{y} ; \mathbf{n}\left(\mathbf{x}_{0}\right)\right) \mathrm{d} \Gamma \mathbf{p}^{+}\left(\mathbf{x}_{0}\right)+\mathbf{f t}_{p u} \\
& -\lim _{\mathbf{x} \rightarrow \mathbf{x}_{0}}\left\{\int_{\Gamma_{\varepsilon}} \mathbf{G}_{p p}(\mathbf{r} ; \mathbf{n}(\mathbf{x}) ; \mathbf{l}(\mathbf{y}))\left[\mathbf{u}(\mathbf{y})-\mathbf{u}\left(\mathbf{x}_{0}\right)-\boldsymbol{\epsilon}\left(\mathbf{x}_{0}\right)\left(\mathbf{y}-\mathbf{x}_{0}\right)\right] \mathrm{d} \Gamma\right\} \\
& -f_{\Gamma_{\varepsilon}} \mathbf{G}_{p p}\left(\mathbf{x}_{0}-\mathbf{y} ; \mathbf{n}(\mathbf{x}) ; \mathbf{l}(\mathbf{y})\right) \mathrm{d} \Gamma \mathbf{u}\left(\mathbf{x}_{0}\right)-f_{\Gamma_{\varepsilon}} \mathbf{G}_{p p}\left(\mathbf{x}_{0}-\mathbf{y} ; \mathbf{n}(\mathbf{x}) ; \mathbf{l}(\mathbf{y})\right) \boldsymbol{\epsilon}\left(\mathbf{x}_{0}\right) \mathbf{y} \mathrm{d} \Gamma \\
& +\mathbf{f t}_{p p}^{0}+\mathbf{f t}_{p p}^{1}
\end{aligned}
$$

Defining with:

$$
\begin{aligned}
& \mathbf{D}_{p p}\left(\mathbf{x}_{0}, \mathbf{n}\left(\mathbf{x}_{0}\right)\right) \stackrel{\text { def }}{=}-\frac{\left(\mathbf{f t}_{p p}^{0}+\mathbf{f t}_{p p}^{1}\right) \otimes \mathbf{p}\left(\mathbf{x}_{0}, \mathbf{n}\left(\mathbf{x}_{0}\right)\right)}{\left\|\mathbf{p}\left(\mathbf{x}_{0}, \mathbf{n}\left(\mathbf{x}_{0}\right)\right)\right\|^{2}} \\
& \mathbf{D}_{p u}\left(\mathbf{x}_{0}, \mathbf{n}\left(\mathbf{x}_{0}\right)\right) \stackrel{\text { def }}{=} \frac{\mathbf{f t}_{p u} \otimes \mathbf{p}\left(\mathbf{x}_{0}, \mathbf{n}\left(\mathbf{x}_{0}\right)\right)}{\left\|\mathbf{p}\left(\mathbf{x}_{0}, \mathbf{n}\left(\mathbf{x}_{0}\right)\right)\right\|^{2}}
\end{aligned}
$$

the thesis is proved by means of eq. (9) and of Proposition 4.2.

Remarks to proposition 4.3

- Equation (20) extends the traction equation (2) on non-smooth boundaries. In fact, it is well known (see e.g. [3] page 18) that on smooth boundaries:

$$
\begin{aligned}
& f_{\Gamma_{\varepsilon}} \mathbf{G}_{p u}(\mathbf{r} ; \mathbf{n}(\mathbf{x})) \mathbf{p}(\mathbf{y}) \mathrm{d} \Gamma_{\mathbf{y}}= \\
& \quad f_{\Gamma_{\varepsilon}^{-}} \mathbf{G}_{p u}(\mathbf{r} ; \mathbf{n}(\mathbf{x})) \mathbf{p}(\mathbf{y}) \mathrm{d} \Gamma_{\mathbf{y}}+f_{\Gamma_{\varepsilon}^{+}} \mathbf{G}_{p u}(\mathbf{r} ; \mathbf{n}(\mathbf{x})) \mathbf{p}(\mathbf{y}) \mathrm{d} \Gamma_{\mathbf{y}}
\end{aligned}
$$


therefore eq. (20) coincides with eq. (2) on smooth boundaries.

It is worth noting in the proof that the logarithmic unbounded term due to the limit to the boundary (14) cancels out with the unbounded counterpart arising from the strongly singular kernel (17). This event gives sense to the equation (20) on non-smooth boundaries, owing to a property of the global (elastic) problem. The finite part formulation (20) should therefore be intended to corners as an extension of equation (2) which may be proved only for smooth boundaries.

- The free term coefficient $\mathbf{D}(\mathbf{x})$ now holds:

$$
\mathbf{D}(\mathbf{x})=\mathbf{I}-\frac{\left(\mathbf{f t}_{p u}+\mathbf{f t}_{p p}^{0}+\mathbf{f t}_{p p}^{1}\right) \otimes \mathbf{p}\left(\mathbf{x}_{0}, \mathbf{n}\left(\mathbf{x}_{0}\right)\right)}{\left\|\mathbf{p}\left(\mathbf{x}_{0}, \mathbf{n}\left(\mathbf{x}_{0}\right)\right)\right\|^{2}}
$$

and it is perfectly defined, in view of Proposition 4.2, as a result of a limit process. It holds obviously

$$
\mathbf{D}(\mathbf{x}) \mathbf{p}(\mathbf{x}, \mathbf{n}(\mathbf{x}))=\mathbf{p}(\mathbf{x}, \mathbf{n}(\mathbf{x}))-\left(\mathbf{f t}_{p u}+\mathbf{f t}_{p p}^{0}+\mathbf{f t}_{p p}^{1}\right)
$$

with $\mathbf{f t}_{p u}+\mathbf{f t}_{p p}^{0}+\mathbf{f t}_{p p}^{1}$ defined by equation (19); by direct substitution, one proves the following corollary:

Proposition 4.4 If $\varphi=0$ then $\mathbf{D}(\mathbf{x}) \mathbf{p}(\mathbf{x}, \mathbf{n}(\mathbf{x}))=\frac{1}{2} \mathbf{p}(\mathbf{x}, \mathbf{n}(\mathbf{x}))$

- In view of the previous remark, $\mathbf{D}(\mathbf{x})$ depends on angle $\varphi$, which is expected, and of the material properties of the body due to the constitutive law (18). The dependence on the elastic constants does not seem to be avoidable, to make use of equation (18) it is necessary to define the existence of limit (19). Similar considerations hold for the dependence on $\boldsymbol{\sigma}$ and on $\mathbf{n}(\mathbf{x})$, in view of the previous remark. At any rate, further investigations are required in order to establish further properties which may be hidden by the formal complexity of (19).

The following proposition seems to be of interest, especially for its numerical implications:

Proposition 4.5 If displacement and stress fields satisfy the constitutive equation (18), then it holds:

$$
\begin{gathered}
f_{\Gamma_{\varepsilon}^{-}} \mathbf{G}_{p u}\left(\mathbf{x}_{0}-\mathbf{y} ; \mathbf{n}\left(\mathbf{x}_{0}\right)\right) \mathrm{d} \Gamma \mathbf{p}^{-}\left(\mathbf{x}_{0}\right)+f_{\Gamma_{\varepsilon}^{+}} \mathbf{G}_{p u}\left(\mathbf{x}_{0}-\mathbf{y} ; \mathbf{n}\left(\mathbf{x}_{0}\right)\right) \mathrm{d} \Gamma \mathbf{p}^{+}\left(\mathbf{x}_{0}\right) \\
-f_{\Gamma_{\varepsilon}} \mathbf{G}_{p p}\left(\mathbf{x}_{0}-\mathbf{y} ; \mathbf{n}(\mathbf{x}) ; \mathbf{l}(\mathbf{y})\right) \boldsymbol{\epsilon}\left(\mathbf{x}_{0}\right) \mathbf{y} \mathrm{d} \Gamma=\mathbf{0}
\end{gathered}
$$


Proof: It was shown in proposition 4.3 that:

$$
\begin{aligned}
& -f_{\Gamma_{\varepsilon}} \mathbf{G}_{p p}\left(\mathbf{x}_{0}-\mathbf{y} ; \mathbf{n}(\mathbf{x}) ; \mathbf{l}(\mathbf{y})\right) \boldsymbol{\epsilon}\left(\mathbf{x}_{0}\right) \mathbf{y} \mathrm{d} \Gamma= \\
& \quad=f_{x_{1}^{-}-\frac{\varepsilon}{2}}^{x_{1}^{-}+\frac{\varepsilon}{2}} \mathbf{G}_{p p}^{-}() r_{1}^{-} \mathrm{d} r_{1} \boldsymbol{\epsilon}^{-}\left(\mathbf{x}_{0}\right) \mathbf{e}_{1}^{-}+\mathbf{A}^{\top} f_{x_{1}^{+}-\frac{\varepsilon}{2}}^{x_{1}^{+}+\frac{\varepsilon}{2}} \mathbf{G}_{p p}^{+}() r_{1}^{+} \mathrm{d} r_{1} \mathbf{A} \boldsymbol{\epsilon}^{-}\left(\mathbf{x}_{0}\right) \mathbf{A}^{\top} \mathbf{e}_{1}^{+} \\
& \quad \stackrel{(\underline{28})}{=} \frac{G}{4 \pi(1-\nu)}\left[\mathbf{A}^{\top}\left(\begin{array}{cc}
n_{2}^{+} & n_{1}^{+} \\
n_{1}^{+} & n_{2}^{+}
\end{array}\right) \mathbf{A} \boldsymbol{\epsilon}^{-}\left(\mathbf{x}_{0}\right) \mathbf{A}^{\top} \mathbf{e}_{1}^{+}-\left(\begin{array}{cc}
n_{2}^{-} & n_{1}^{-} \\
n_{1}^{-} & n_{2}^{-}
\end{array}\right) \boldsymbol{\epsilon}^{-}\left(\mathbf{x}_{0}\right) \mathbf{e}_{1}^{-}\right] \log \|\varepsilon\| \\
& \quad=\mathbf{l}_{p p}^{2}(\varphi, \mathbf{n}, \boldsymbol{\epsilon}, G, \nu) \log \|\varepsilon\|
\end{aligned}
$$

where equation (28) has been used. On the other hand,

$$
\begin{aligned}
& f_{\Gamma_{\varepsilon}^{-}} \mathbf{G}_{p u}\left(\mathbf{x}_{0}-\mathbf{y} ; \mathbf{n}\left(\mathbf{x}_{0}\right)\right) \mathrm{d} \Gamma \mathbf{p}^{-}\left(\mathbf{x}_{0}\right)+f_{\Gamma_{\varepsilon}^{+}} \mathbf{G}_{p u}\left(\mathbf{x}_{0}-\mathbf{y} ; \mathbf{n}\left(\mathbf{x}_{0}\right)\right) \mathrm{d} \Gamma \mathbf{p}^{+}\left(\mathbf{x}_{0}\right)= \\
& f_{-\varepsilon}^{0} \mathbf{G}_{p u}^{-} \mathrm{d} y_{1} \boldsymbol{\sigma}^{-}\left(\mathbf{x}_{0}\right) \mathbf{e}_{2}^{-}+\mathbf{A}^{\top} f_{0}^{\varepsilon} \mathbf{G}_{p u}^{+} \mathrm{d} y_{1} \mathbf{A} \boldsymbol{\sigma}^{-}\left(\mathbf{x}_{0}\right) \mathbf{A}^{\top} \mathbf{l}^{+}= \\
& \frac{1}{4 \pi(1-\nu)}\left[\left(\begin{array}{cc}
n_{1}^{-}(-3+2 \nu) & n_{2}^{-}(-1+2 \nu) \\
n_{2}^{-}(1-2 \nu) & n_{1}^{-}(-1+2 \nu)
\end{array}\right) \boldsymbol{\sigma}^{-}\left(\mathbf{x}_{0}\right) \mathbf{e}_{2}^{-}+\right. \\
& \left.-\mathbf{A}^{\top}\left(\begin{array}{cc}
n_{1}^{+}(-3+2 \nu) & n_{2}^{+}(-1+2 \nu) \\
n_{2}^{+}(1-2 \nu) & n_{1}^{+}(-1+2 \nu)
\end{array}\right) \mathbf{A} \boldsymbol{\sigma}^{-}\left(\mathbf{x}_{0}\right) \mathbf{A}^{\top} \mathbf{l}^{+}\right] \log \|\varepsilon\| \\
& =\mathbf{l}_{p u}(\varphi, \mathbf{n}, \boldsymbol{\sigma}, \nu) \log \|\varepsilon\|
\end{aligned}
$$

It holds however:

$$
\begin{gathered}
\mathbf{l}_{p u}(\varphi, \mathbf{n}, \boldsymbol{\sigma}, \nu)=-\mathbf{l}_{p p}^{2}(\varphi, \mathbf{n}, \boldsymbol{\epsilon}, G, \nu)=\frac{1}{16 \pi(-1+v)} \\
\left\{\begin{array}{c}
\sin (\varphi)\left(\left(2 n_{2} \sigma_{21}+n_{1}\left(\sigma_{22}+4 \sigma_{22} \nu+\sigma_{11}(-5+4 \nu)\right)\right) \cos (\varphi)+\right. \\
\left(2 n_{2} \sigma_{21}+n_{1}\left(-\sigma_{11}+\sigma_{22}\right)\right) \cos (3 \varphi)+ \\
2\left(4 n_{1} \sigma_{21}-2 n_{2}\left(\sigma_{11}(-1+\nu)+\sigma_{22} \nu\right)+\right. \\
\left.\left.\left(2 n_{1} \sigma_{21}+n_{2}\left(\sigma_{11}-\sigma_{22}\right)\right) \cos (2 \varphi)\right) \sin (\varphi)\right) \\
\sin (\varphi)\left(\left(2 n_{1} \sigma_{21}+n_{2}\left(\sigma_{11}+3 \sigma_{22}-4 \sigma_{11} \nu-4 \sigma_{22} \nu\right)\right) \cos (\varphi)+\right. \\
\left(2 n_{1} \sigma_{21}+n_{2}\left(\sigma_{11}-\sigma_{22}\right)\right) \cos (3 \varphi)- \\
2\left(2 n_{1}\left(\sigma_{11}(-1+\nu)+\sigma_{22} \nu\right)+\right. \\
\left.\left.\left.\left(2 n_{2} \sigma_{21}+n_{1}\left(-\sigma_{11}+\sigma_{22}\right)\right) \cos (2 \varphi)\right) \sin (\varphi)\right)\right\}
\end{array}\right.
\end{gathered}
$$

and the thesis follows.

Remarks to proposition 4.5 
- Of course proposition 4.5 applies to smooth boundaries: in that case however it is trivial, for being

$$
\begin{aligned}
f_{\Gamma_{\varepsilon}} \mathbf{G}_{p u}\left(\mathbf{x}_{0}-\mathbf{y} ; \mathbf{n}\left(\mathbf{x}_{0}\right)\right) \mathrm{d} \Gamma \mathbf{p}^{-}\left(\mathbf{x}_{0}\right)= \\
\quad f_{\Gamma_{\varepsilon}} \mathbf{G}_{p p}\left(\mathbf{x}_{0}-\mathbf{y} ; \mathbf{n}(\mathbf{x}) ; \mathbf{l}(\mathbf{y})\right) \boldsymbol{\epsilon}\left(\mathbf{x}_{0}\right) \mathbf{y} \mathrm{d} \Gamma=\mathbf{0}
\end{aligned}
$$

- The evaluation of equation (2) is not a trivial task, because of the involved singularities, especially for the hypersingular kernel. Several techniques, collectable in three principal groups, have been proposed for their evaluation: (i) regularization techniques, (ii) numerical integrations, (iii) analytical integrations.

Numerical methods for the evaluation of the CPV were proposed first in [4]. There is nowadays extensive literature on this subject (see, among others, [5]-[6]). A huge amount of literature concerns the numerical evaluation of hypersingular integrals: among others, [7]-[8]. In almost all numerical schemes, the evaluation of the terms of identity (23) is the major time-consuming task. For this reason, schemes that may take into account of identity (23) seem to be of interest in the framework of the hypersingular BEM.

- Identity (23), as well as all results that have been obtained by making use of the constitutive law (18), holds for the exact solution fields $\mathbf{u}(\mathbf{y}), \mathbf{p}(\mathbf{y})$ but generally not for the approximated fields that pertain to the numerical solution. One must take this problem into account, as well as the unboundedness due to Proposition 4.1, in every post-processing task.

\section{A numerical benchmark.}

In this section, numerical results on the stress tensor evaluation are presented, to confirm the previous analysis. Consider the square domain of figure 3, with sides of length 2. Two different load sets are considered. In the spirit of an inverse method, a radial displacement field $\mathbf{u}(\mathbf{x})=\left(x_{1}, x_{2}\right)$ is firstly considered as the solution of a (plane strain) elastic problem; the corresponding plane components of the stress tensor are $\boldsymbol{\sigma}(\mathbf{x})=E /((1+\nu)(1-2 \nu)) \mathbf{I}$. The lower horizontal side is constrained, whereas the remaining sides are loaded with a normal traction of intensity $p=E /((1+\nu)(1-2 \nu))$.

In the second load set, the lower horizontal side is constrained by $\overline{\mathbf{u}}(\mathbf{y})=\mathbf{0}$. The upper horizontal side is subjected to the vertical load $\overline{\mathbf{p}}(\mathbf{y})=\left(1+0.02 x_{2}\right)(\lambda+$ $2 G) \mathbf{e}_{2}$, while the two vertical sides are loaded by $\overline{\mathbf{p}}(\mathbf{y})=\left(1+0.02 x_{2}\right) \lambda \mathbf{l}(\mathbf{y})$, denoting with $\lambda$ and $G$ the Lamè constants and by $\mathbf{l}(\mathbf{y})$ the outward normal. The analytical solution of the problem reads:

$$
\mathbf{u}(\mathbf{y})=x_{2}\left(1+0.01 x_{2}\right) \mathbf{e}_{2}
$$




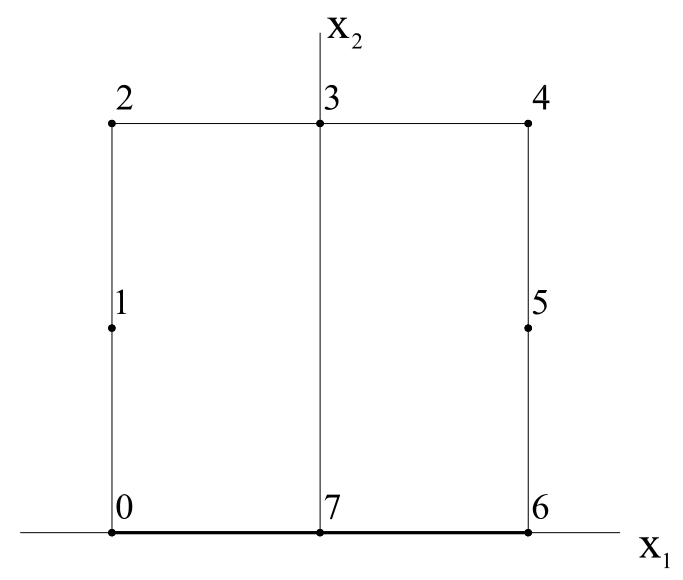

Figure 3: Geometry and discretization by means of 16 equal linear boundary elements.

$$
\boldsymbol{\sigma}(\mathbf{y})=\left(1+0.02 x_{2}\right)\left(\begin{array}{ccc}
\lambda & 0 & 0 \\
0 & \lambda+2 G & 0 \\
0 & 0 & \lambda
\end{array}\right)
$$

Having approximated the problem via the SGBEM as well as via the collocation method, owing to the discretization of figure 3 making use of linear boundary elements, the stress tensor has been evaluated at a sequence of points $\mathbf{x}_{n} \rightarrow \mathbf{x}_{\infty}$ as a post processing task and numerical comparisons in terms of accuracy are plot.

With regard to the first load set, the linear approximation coincides with the exact solution: accordingly, no numerical errors are expected as the point $\mathbf{x}_{n} \in \Omega$ moves to the point $\mathbf{x}_{\infty} \in \Gamma$. This is confirmed by the stress tensor analysis on the set $\mathbf{x}_{n}=\left\{1-0.1^{n}, 0.5\right\}$ : in fact, for being $\mathbf{x}_{\infty}=\{1,0.5\}$ inside a boundary element, proposition 2.2 of the companion paper [1] apply. On the contrary, by considering the set $\mathbf{x}_{n}=\left\{\left(\left(1-0.1^{n}\right), 2\left(1-0.1^{n}\right)\right)\right\}$ converging at a corner, numerical errors come into play, as shown in figure 4 . Such an error, which shows a linear behavior, is due to the numerical cancellation. In fact, because the linear approximation coincides with the exact solution, proposition 4.1 holds, but the computer code shows to be not robust enough to take into account of the cancellation of logarithmic terms of equations (14)-(17).

Considering the second load set, the linear numerical solution does not comply with the quadratic nature of the displacement field and approximations are therefore introduced. Assuming the set $\mathbf{x}_{n}=\left\{\left(\left(1-0.1^{n}\right), 2\left(1-0.1^{n}\right)\right)\right\}$ converging at a corner, numerical errors come again into play, as shown in figure 4 , but they have a very different nature and behavior. They are in fact due to the non applicability of proposition 4.1, because the approximated stress and displacement field do not satisfy the constitutive law (18). As a consequence,

$$
\mathbf{l}_{p p}^{1}(\varphi, \mathbf{n}, \boldsymbol{\epsilon}(\boldsymbol{\sigma}), G, \nu) \neq \mathbf{l}_{p u}^{1}(\varphi, \mathbf{n}, \boldsymbol{\sigma}, G, \nu)
$$




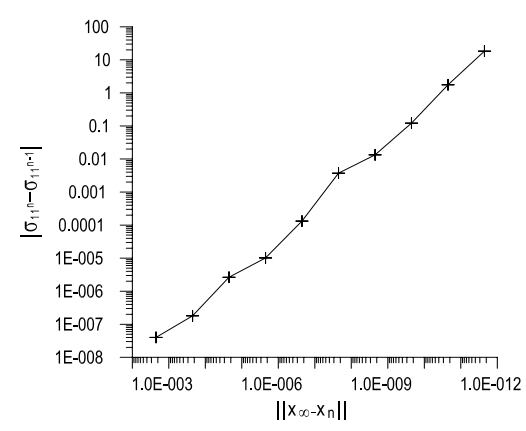

a)

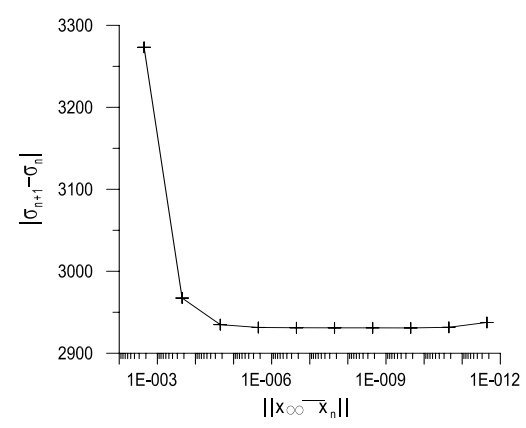

b)

Figure 4: Error at a corner: a) first load set, b) second load set

and a logarithmic unbounded term is expected in the limit process $\mathbf{x}_{n} \rightarrow \mathbf{x}_{\infty}$. Figure 4 shows this error, which takes the form:

$$
\boldsymbol{\sigma}_{11}\left(\mathbf{x}_{n+1}\right)-\boldsymbol{\sigma}_{11}\left(\mathbf{x}_{n+1}\right) \sim 2930
$$

on the set $\mathbf{x}_{n}=\left\{\left(\left(1-0.1^{n}\right), 2\left(1-0.1^{n}\right)\right)\right\} \quad n=1,2, \ldots, 12$. The computer code shows to be not robust enough to increase further the value of $n$, however the numerical analysis is in perfect agreement with the theory in previous section, thus confirming that if the approximated stress and displacement field do not satisfy the constitutive law (18) the stress tensor evaluation at a corner is not feasible.

\section{Concluding remarks.}

In the present work the hypersingular formulation for boundary stress evaluation has been revised along boundaries that present a corner.

When the boundary is smooth, it is possible to distinguish and determine the hypersingular and the strongly singular kernels free term contributions, as was done in the companion paper [1]. In the presence of corners, on the contrary, the limit to the boundary of each (hyper)singular integral is not bounded and a logarithmic unbounded term comes into play. Only by neglecting such a term, is it possible to recognize "candidate" factors which act as free terms; unfortunately such terms cannot be defined by a limit process, for they depend on the direction by which the limit process itself is taken.

In conclusion, it is questionable if the hypersingular finite part formulation for boundary stress evaluation (2) makes sense, as the limit process of the integral of each singular kernel neither produces Hadamard's finite parts nor Cauchy's principal values. However, the intimate relationship between hypersingular boundary integral equations, Hadamard's finite part, and Cauchy's principal value is again confirmed even in the presence of corners. In fact, it has been proved that not 
only the unbounded terms cancel out in the sum of hypersingular and strongly singular contributions, but the sum of the free term candidates is perfectly defined by a limit process. Accordingly, the finite part formulation for boundary stress evaluation makes sense by a global property of the elastic operator and not by a property of each kernel. Such a conclusion was conjectured by various authors but, to the best of my knowledge, it was never proved so far.

To emphasize that equation (2) is justified only for the global problem, it has been proved to apply only to fields that comply with the constitutive law (18). For all fields that do not fulfill the constitutive law, equation (2) may not hold. For instance, equation (2) leads to the boundary element method by considering discrete approximation for displacement and traction fields: for the discrete traction equation on the boundary, it has been proved that the free term coefficient on smooth boundaries does not hold $\frac{1}{2} \mathbf{I}$ (see [1]) and that the free term coefficient is no longer defined in the presence of corners.

As a complementary result in the present work, it was shown that Hadamard's finite parts (with respect to the linear terms) and Cauchy's principal values cancel themselves out (see Proposition 4.5). Because the computational cost of their evaluation is high, such a property may be of interest for the numerical implementation of the BEM.

As a major drawback of the proposed methodology, analytical integrations reveal to be formally very complex. As a consequence, their manipulation is not straightforward and can be done in practice only with the help of a system by doing mathematics by computer. In this way, it is not always easy to figure out whether (unexpected) results are due to the computer code representation or wether they are due to the real nature of the solution (see e.g. the dependence of the free term coefficient on the material properties of the body).

The present work is preliminary to the analysis of engineering problems, for which the stress tensor along the boundary is a fundamental item. In particular, problems of fracture initiation, propagation and bifurcation in soil-structure interactions [9], in composite [10] and biological materials and tissues are under investigation [11].

\section{Acknowledgements}

Part of the present work was performed in the first semester of 2008 at the Oak Ridge National Laboratory: I am grateful to Professor L.J.Gray for the opportunity of visiting and working with him. The support of the Italian Ministry of University and Research (MIUR) under grant ex 60\% - 2007: "Analisi non lineari

nella meccanica dei continui e della frattura mediante il metodo degli elementi al contorno" is gratefully acknowledged. 


\section{References}

[1] A. Salvadori. Hypersingular formulation for boundary stress evaluation revisited. part 1: Smooth boundaries. In press on the "Electronic Journal of Boundary Elements", 2008.

[2] A. Salvadori. Analytical integrations in 2D BEM elasticity. Int. J. Numer. Methods Eng., 53(7):1695-1719, 2002.

[3] A.H. Zemanian. Distribution theory and transform analysis. Dover, 1987.

[4] H.R. Kutt. The numerical evaluation of principal value integrals by finite-part integration. Numer. Math., 24:205-210, 1975.

[5] G. Monegato. The numerical evaluation of hypersingular integrals. J. Comput. Appl. Math., 1994.

[6] D. Elliott and D.F. Paget. Gauss type quadrature rules for Cauchy principal value integrals. Mathematics of Computation, 33(145):301-309, 1979.

[7] M. Diligenti and G. Monegato. Finite-part integrals, their occurence and computation. Rend. Circ. Mat. Palermo, 33(II):39-61, 1993.

[8] C.Y. Hui and D. Shia. Evaluations of hypersingular integrals using gaussian quadrature. Int. J. Numer. Methods Eng., 44:205-214, 1999.

[9] S.T. Slowik, J.M. Chandra Kishen, and V.E. Saouma. Mixed mode fracture of cementitious interfaces. Part 1: experimental results. Engineering Fracture Mechanics. Mech., 60:83-94, 1998.

[10] S.T. Smith and J.G. Teng. Interfacial stresses in plated beams. Engineering Structures, 23:857-871, 2001.

[11] A. Salvadori. A symmetric boundary integral formulation for cohesive interface problems. Computational Mechanics, 32(4-6):381-391, 2003.

\section{Appendix 1 - Hypersingular kernel integration at a corner.}

This appendix focuses on the mathematical passages required by the free term analysis of the hypersingular integrals (10) and (11). By assuming $\mathbf{x} \in \Omega$ consider the two integrals:

$$
\begin{array}{lr}
\int_{-\varepsilon}^{0} \mathbf{G}_{p p}^{-} \mathrm{d} y_{1} \mathbf{u}^{-}\left(\mathbf{x}_{0}\right)+\int_{-\varepsilon}^{0} \mathbf{G}_{p p}^{-} y_{1} \mathrm{~d} y_{1}\left(\boldsymbol{\epsilon}^{-}\left(\mathbf{x}_{0}\right) \mathbf{e}_{1}^{-}+\kappa^{-} \mathbf{u}^{-}\left(\mathbf{x}_{0}\right)\right) & \text { in } \mathcal{A} 24) \\
\int_{0}^{\varepsilon} \mathbf{G}_{p p}^{+} \mathrm{d} y_{1} \mathbf{u}^{+}\left(\mathbf{x}_{0}\right)+\int_{0}^{\varepsilon} \mathbf{G}_{p p}^{+} y_{1} \mathrm{~d} y_{1}\left(\boldsymbol{\epsilon}^{+}\left(\mathbf{x}_{0}\right) \mathbf{e}_{1}^{+}+\kappa^{+} \mathbf{u}^{+}\left(\mathbf{x}_{0}\right)\right) & \text { in } \mathcal{L}(25)
\end{array}
$$



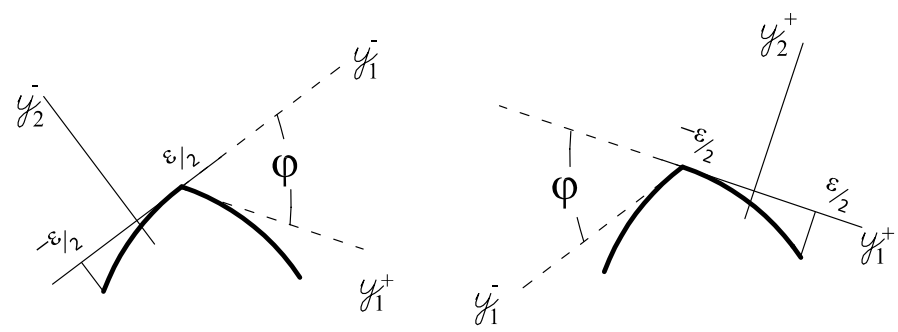

Figure 5: Local reference for analytical integration.

where $\mathbf{e}_{1}^{+}$denotes, see figure 5 , the unit vector associated to the $y_{1}^{+}$-axis. In order to sum all contributions, the same coordinate system must be set. With reference to figure 5 the matrix

$$
\mathbf{A}=\left(\begin{array}{cc}
\cos \varphi & -\sin \varphi \\
\sin \varphi & \cos \varphi
\end{array}\right)
$$

governs the reference change between $\mathcal{L}^{-}$and $\mathcal{L}^{+}$: accordingly $X^{-}=\mathbf{A}^{\top} X^{+} \mathbf{A}$ for any tensor $X^{+}$in the local reference $\mathcal{L}^{+}$and $x^{-}=\mathbf{A}^{\top} x^{+}$for any vector $x^{+}$in the local reference $\mathcal{L}^{+}$. For being

$$
\begin{gathered}
\mathbf{p}^{-}=\mathbf{A}^{\top} \mathbf{p}^{+} \sim \mathbf{A}^{\top} \mathbf{G}_{p p}^{+} \mathbf{u}^{+}\left(\mathbf{x}_{0}\right)=\mathbf{A}^{\top} \mathbf{G}_{p p}^{+} \mathbf{A} \mathbf{A}^{\top} \mathbf{u}^{+}\left(\mathbf{x}_{0}\right)=\mathbf{A}^{\top} \mathbf{G}_{p p}^{+} \mathbf{A} \mathbf{u}^{-}\left(\mathbf{x}_{0}\right) \\
\mathbf{p}^{-}=\mathbf{A}^{\top} \mathbf{p}^{+} \sim \mathbf{A}^{\top} \mathbf{G}_{p p}^{+} \boldsymbol{\epsilon}^{+}\left(\mathbf{x}_{0}\right) \mathbf{e}_{1}^{+}=\mathbf{A}^{\top} \mathbf{G}_{p p}^{+} \mathbf{A} \mathbf{A}^{\top} \boldsymbol{\epsilon}^{+}\left(\mathbf{x}_{0}\right) \mathbf{A} \mathbf{A}^{\top} \mathbf{e}_{1}^{+} \\
=\mathbf{A}^{\top} \mathbf{G}_{p p}^{+} \mathbf{A} \boldsymbol{\epsilon}^{-}\left(\mathbf{x}_{0}\right) \mathbf{A}^{\top} \mathbf{e}_{1}^{+}
\end{gathered}
$$

it comes out that the sum of integrals (24) and (25) holds:

$$
\begin{aligned}
& {\left[\int_{-\varepsilon}^{0} \mathbf{G}_{p p}^{-}\left(1+\kappa^{-} y_{1}\right) \mathrm{d} y_{1}+\mathbf{A}^{\top} \int_{0}^{\varepsilon} \mathbf{G}_{p p}^{+}\left(1+\kappa^{+} y_{1}\right) \mathrm{d} y_{1} \mathbf{A}\right] \mathbf{u}^{-}\left(\mathbf{x}_{0}\right)+} \\
& \int_{-\varepsilon}^{0} \mathbf{G}_{p p}^{-} y_{1} \mathrm{~d} y_{1} \boldsymbol{\epsilon}^{-}\left(\mathbf{x}_{0}\right) \mathbf{e}_{1}^{-}+\mathbf{A}^{\top} \int_{0}^{\varepsilon} \mathbf{G}_{p p}^{+} y_{1} \mathrm{~d} y_{1} \mathbf{A} \boldsymbol{\epsilon}^{-}\left(\mathbf{x}_{0}\right) \mathbf{A}^{\top} \mathbf{e}_{1}^{+}
\end{aligned}
$$

in reference $\mathcal{L}^{-}$.

\subsection{Constant term analysis}

With reference to figure 5 and $\mathbf{x} \in \Omega$, the integral

$$
\int_{0}^{\varepsilon} \mathbf{G}_{p p}^{+} \mathrm{d} y_{1}^{+}=\int_{-\frac{\varepsilon}{2}}^{\frac{\varepsilon}{2}} \mathbf{G}_{p p}^{+} \mathrm{d} y_{1}^{+}=\int_{x_{1}^{+}-\frac{\varepsilon}{2}}^{x_{1}^{+}+\frac{\varepsilon}{2}} \mathbf{G}_{p p}^{+} \mathrm{d} r_{1}^{+}=\frac{1}{r^{2}}+\mathbf{S}_{p p}^{(0)}+\left.\frac{1}{r^{4}}{ }^{+} \mathbf{H}_{p p}^{(0)}\right|_{r_{1}^{+}=x_{1}^{+}-\frac{\varepsilon}{2}} ^{r_{1}^{+}=x_{1}^{+}+\frac{\varepsilon}{2}}
$$

has been solved in [2] by means of the variable change $\mathbf{r}^{+}=\mathbf{x}^{+}-\mathbf{y}^{+}$; matrices $\mathbf{S}_{p p}^{(0)}, \mathbf{H}_{p p}^{(0)}$ follow in the local reference $\mathcal{L}^{+}$:

$$
{ }^{+} \mathbf{S}_{p p}^{(0)}=-\frac{G}{2 \pi(1-\nu)}\left(\begin{array}{cc}
n_{2}^{+} r_{1}^{+}-3 n_{1}^{+} r_{2}^{+} & n_{1}^{+} r_{1}^{+}+n_{2}^{+} r_{2}^{+} \\
n_{1}^{+} r_{1}^{+}+n_{2}^{+} r_{2}^{+} & n_{2}^{+} r_{1}^{+}+n_{1}^{+} r_{2}^{+}
\end{array}\right)
$$




$$
{ }^{+} \mathbf{H}_{p p}^{(0)}=-\frac{G\left(r_{2}^{+}\right)^{2}}{\pi(1-\nu)}\left(\begin{array}{cc}
-n_{2}^{+} r_{1}^{+}+n_{1}^{+} r_{2}^{+} & -n_{1}^{+} r_{1}^{+}-n_{2}^{+} r_{2}^{+} \\
-n_{1}^{+} r_{1}^{+}-n_{2}^{+} r_{2}^{+} & n_{2}^{+} r_{1}^{+}-n_{1}^{+} r_{2}^{+}
\end{array}\right)
$$

A similar result holds for the integral

$$
\int_{-\varepsilon}^{0} \mathbf{G}_{p p}^{-} \mathrm{d} y_{1}^{-}=\int_{-\frac{\varepsilon}{2}}^{\frac{\varepsilon}{2}} \mathbf{G}_{p p}^{-} \mathrm{d} y_{1}^{-}=\int_{x_{1}^{-}-\frac{\varepsilon}{2}}^{x_{1}^{-}+\frac{\varepsilon}{2}} \mathbf{G}_{p p}^{-} \mathrm{d} r_{1}^{-}=\frac{1}{r^{2}}-\mathbf{S}_{p p}^{(0)}+\left.\frac{1}{r^{4}}{ }^{-} \mathbf{H}_{p p}^{(0)}\right|_{r_{1}^{+}=x_{1}^{+}-\frac{\varepsilon}{2}} ^{r_{1}^{+}=x_{1}^{+}+\frac{\varepsilon}{2}}
$$

The limit to the boundary $\Omega \ni \mathbf{x} \rightarrow \mathbf{x}_{0} \in \Gamma$ is taken by means of the norm $\left\|\mathbf{x}-\mathbf{x}_{0}\right\| \rightarrow 0$ and of a direction, selected through the angle $\theta$ as in figure 6 . It is
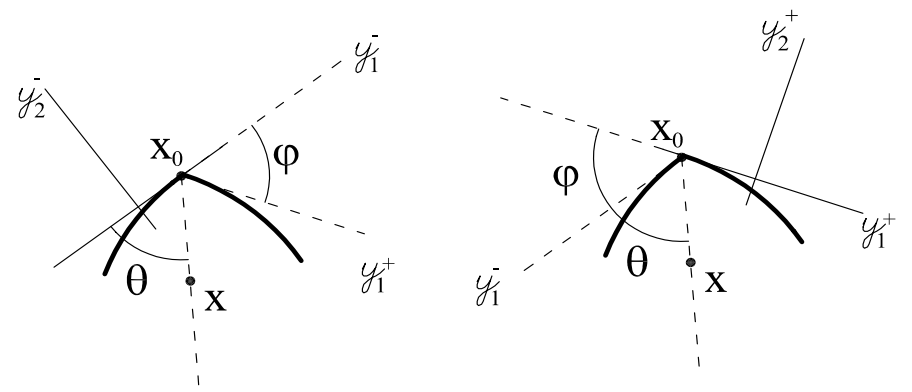

Figure 6: Limit process $\Omega \ni \mathbf{x} \rightarrow \mathbf{x}_{0} \in \Gamma$.

worth noting that the limit

$$
\lim _{\mathbf{x} \rightarrow \mathbf{x}_{0}} \int_{0}^{\varepsilon} \mathbf{G}_{p p}^{+} \mathrm{d} y_{1}^{+}=f_{0}^{\varepsilon} \mathbf{G}_{p p}^{+} \mathrm{d} y_{1}^{+}+\mathbf{A} \frac{G}{2 \pi(1-\nu)} \mathbf{W}(\theta) \mathbf{A}^{\top} \frac{1}{\left\|\mathbf{x}-\mathbf{x}_{0}\right\|}
$$

with:

$\mathbf{W}(\theta) \stackrel{\text { def }}{=}\left(\begin{array}{cc}\frac{n_{2}(\cos (\theta)+\cos (3 \theta))-n_{1}(3 \sin (\theta)+\sin (3 \theta))}{2} & \cos (2 \theta)\left(n_{1} \cos (\theta)+n_{2} \sin (\theta)\right) \\ \cos (2 \theta)\left(n_{1} \cos (\theta)+n_{2} \sin (\theta)\right) & \frac{3 n_{2}(\cos (\theta)-\cos (3 \theta))+n_{1}(-\sin (\theta)+\sin (3 \theta))}{2}\end{array}\right)$

is not well defined. The "inconsistent" terms however cancel out in the sum, so that

$$
\lim _{\mathbf{x} \rightarrow \mathbf{x}_{0}}\left[\int_{-\varepsilon}^{0} \mathbf{G}_{p p}^{-} \mathrm{d} y_{1}+\mathbf{A}^{\top} \int_{0}^{\varepsilon} \mathbf{G}_{p p}^{+} \mathrm{d} y_{1} \mathbf{A}\right]=f_{-\varepsilon}^{0} \mathbf{G}_{p p}^{-} \mathrm{d} y_{1}+\mathbf{A}^{\top} f_{0}^{\varepsilon} \mathbf{G}_{p p}^{+} \mathrm{d} y_{1} \mathbf{A}
$$

As a consequence, no free terms arise from the constant hypersingular integral. Furthermore, even in the presence of a corner, the HFP is the outcome of a limit process for the hypersingular kernel applied to a constant term.

\subsection{Linear term analysis}

By means of the variable change $\mathbf{r}=\mathbf{x}-\mathbf{y}$, it is straightforward to split the linear hypersingular integral in a constant and a linear part with respect to the variable 
$r_{1}$ :

$$
\int_{0}^{\varepsilon} \mathbf{G}_{p p}^{+} y_{1}^{+} \mathrm{d} y_{1}^{+}=\int_{x_{1}^{+}-\frac{\varepsilon}{2}}^{x_{1}^{+}+\frac{\varepsilon}{2}} \mathbf{G}_{p p}^{+} \mathrm{d} r_{1}^{+}\left(x_{1}^{+}+\frac{\varepsilon}{2}\right)-\int_{x_{1}^{+}-\frac{\varepsilon}{2}}^{x_{1}^{+}+\frac{\varepsilon}{2}} \mathbf{G}_{p p}^{+} r_{1}^{+} \mathrm{d} r_{1}^{+}
$$

The free term analysis for the constant part being already performed, denote by:

$$
\begin{aligned}
\lim _{\mathbf{x} \rightarrow \mathbf{x}_{0}}\left\{\left[\frac{\mathbf{S}_{p p}^{(0)}}{r^{2}}\right]_{x_{1}^{-}-\frac{\varepsilon}{2}}^{x_{1}^{-}+\frac{\varepsilon}{2}}\left(x_{1}^{-}-\frac{\varepsilon}{2}\right) \boldsymbol{\epsilon}^{-}\left(\mathbf{x}_{0}\right) \mathbf{e}_{1}^{-}\right. \\
\left.+\left[\frac{\mathbf{A}^{\top} \mathbf{S}_{p p}^{(0)} \mathbf{A}}{r^{2}}\right]_{x_{1}^{+}\left(\mathbf{x}^{-}\right)-\frac{\varepsilon}{2}}^{x_{1}^{+}\left(\mathbf{x}^{-}\right)+\frac{\varepsilon}{2}}\left(x_{1}^{+}+\frac{\varepsilon}{2}\right) \boldsymbol{\epsilon}^{-}\left(\mathbf{x}_{0}\right) \mathbf{A}^{\top} \mathbf{e}_{1}^{+}\right\}=-\mathbf{s}_{p p}^{0}(\theta, \varphi, \mathbf{n}, \boldsymbol{\epsilon}, G, \nu) \\
\lim _{\mathbf{x} \rightarrow \mathbf{x}_{0}}\left\{\left[\frac{\mathbf{H}_{p p}^{(0)}}{r^{4}}\right]_{x_{1}^{-}-\frac{\varepsilon}{2}}^{x_{1}^{-}+\frac{\varepsilon}{2}}\left(x_{1}^{-}-\frac{\varepsilon}{2}\right) \boldsymbol{\epsilon}^{-}\left(\mathbf{x}_{0}\right) \mathbf{e}_{1}^{-}\right. \\
\left.+\left[\frac{\mathbf{A}^{\top} \mathbf{H}_{p p}^{(0)} \mathbf{A}}{r^{4}}\right]_{x_{1}^{+}\left(\mathbf{x}^{-}\right)-\frac{\varepsilon}{2}}^{x_{1}^{+}\left(\mathbf{x}^{-}\right)+\frac{\varepsilon}{2}}\left(x_{1}^{+}+\frac{\varepsilon}{2}\right) \boldsymbol{\epsilon}^{-}\left(\mathbf{x}_{0}\right) \mathbf{A}^{\top} \mathbf{e}_{1}^{+}\right\}=-\mathbf{h}_{p p}^{0}(\theta, \varphi, \mathbf{n}, \boldsymbol{\epsilon}, G, \nu)
\end{aligned}
$$

where $\varphi$ is the angle between the normals $\mathbf{l}^{+}$and $\mathbf{l}^{-}$as in figure $6, \mathbf{n}$ is the selected direction, $G$ and $\nu$ are elastic properties of the body. ${ }^{1}$ Functions $\mathbf{s}_{p p}^{0}$ and $\mathbf{h}_{p p}^{0}$, being independent on $\varepsilon$, act as free terms. It is worth noting that both vanish at $\varphi=0$, as expected from the free term analysis on smooth boundaries.

By comparison with equation (26), it is straightforward to get

$$
\begin{gathered}
\lim _{\mathbf{x} \rightarrow \mathbf{x}_{0}}\left\{\int_{x_{1}^{-}-\frac{\varepsilon}{2}}^{x_{1}^{-}+\frac{\varepsilon}{2}} \mathbf{G}_{p p}^{-} \mathrm{d} r_{1}\left(x_{1}^{-}-\frac{\varepsilon}{2}\right) \boldsymbol{\epsilon}^{-}\left(\mathbf{x}_{0}\right) \mathbf{e}_{1}^{-}+\right. \\
\left.\mathbf{A}^{\top} \int_{x_{1}^{+}-\frac{\varepsilon}{2}}^{x_{1}^{+}+\frac{\varepsilon}{2}} \mathbf{G}_{p p}^{+} \mathrm{d} r_{1}\left(x_{1}^{+}+\frac{\varepsilon}{2}\right) \mathbf{A} \boldsymbol{\epsilon}^{-}\left(\mathbf{x}_{0}\right) \mathbf{A}^{\top} \mathbf{e}_{1}^{+}\right\}= \\
=\frac{G}{2 \pi(1-\nu)} \mathbf{W}(\theta) \boldsymbol{\epsilon}^{-}\left(\mathbf{x}_{0}\right) \lim _{\mathbf{x} \rightarrow \mathbf{x}_{0}}\left\{-\frac{\left(x_{1}^{-}-\frac{\varepsilon}{2}\right)}{\left\|\mathbf{x}-\mathbf{x}_{0}\right\|} \mathbf{e}_{1}^{-}+\frac{\left(x_{1}^{+}+\frac{\varepsilon}{2}\right)}{\left\|\mathbf{x}-\mathbf{x}_{0}\right\|} \mathbf{A}^{\top} \mathbf{e}_{1}^{+}\right\} \\
=-\mathbf{s}_{p p}^{0}-\mathbf{h}_{p p}^{0}
\end{gathered}
$$

With regard to the linear part, in [2] it has been proved that when $\mathbf{x} \in \Omega$ :

$\int_{x_{1}^{+}-\frac{\varepsilon}{2}}^{x_{1}^{+}+\frac{\varepsilon}{2}} \mathbf{G}_{p p}^{+} r_{1}^{+} \mathrm{d} r_{1}^{+}=\log \left\|\mathbf{r}^{+}\right\| \mathbf{L}_{p p}^{(1)}+\arctan \left(\frac{r_{1}^{+}}{x_{2}^{+}}\right) \mathbf{A}_{p p}^{(1)}+\frac{1}{r^{2}} \mathbf{S}_{p p}^{(1)}+\left.\frac{1}{r^{4}} \mathbf{H}_{p p}^{(1)}\right|_{r_{1}^{+}=x_{1}^{+}-\frac{\varepsilon}{2}} ^{r_{1}^{+}=x_{1}^{+}+\frac{\varepsilon}{2}}$

where $\mathbf{L}_{p p}^{(1)}, \mathbf{A}_{p p}^{(1)}, \mathbf{S}_{p p}^{(1)}, \mathbf{H}_{p p}^{(1)}$ are the following matrices in the local reference $\mathcal{L}^{+}$:

$$
\mathbf{L}_{p p}^{(1)}=\frac{G}{2 \pi(1-\nu)}\left(\begin{array}{cc}
n_{2}^{+} & n_{1}^{+} \\
n_{1}^{+} & n_{2}^{+}
\end{array}\right) \quad \mathbf{A}_{p p}^{(1)}=-\frac{G}{\pi(1-\nu)}\left(\begin{array}{cc}
n_{1} & 0 \\
0 & 0
\end{array}\right)
$$

\footnotetext{
${ }^{1}$ Note that the limits above do not exist, for they depend on the direction $\theta$ by which $\mathbf{x} \rightarrow \mathbf{x}_{0}$ (see figure 6); accordingly $\mathbf{s}_{p p}^{0}, \mathbf{h}_{p p}^{0}$ are but notations, not definitions.
} 


$$
\begin{aligned}
\mathbf{S}_{p p}^{(1)} & =-\frac{G r_{2}^{+}}{\pi(1-\nu)}\left(\begin{array}{cc}
-2\left(n_{1}^{+} r_{1}^{+}+n_{2}^{+} r_{2}^{+}\right) & n_{2}^{+} r_{1}^{+}-2 n_{1}^{+} r_{2}^{+} \\
n_{2}^{+} r_{1}^{+}-2 n_{1}^{+} r_{2}^{+} & n_{1}^{+} r_{1}^{+}+n_{2}^{+} r_{2}^{+}
\end{array}\right) \\
\mathbf{H}_{p p}^{(1)} & =-\frac{G\left(r_{2}^{+}\right)^{3}}{\pi(1-\nu)}\left(\begin{array}{cc}
n_{1}^{+} r_{1}^{+}+n_{2}^{+} r_{2}^{+} & n_{1}^{+} r_{2}^{+}-n_{2}^{+} r_{1}^{+} \\
n_{1}^{+} r_{2}^{+}-n_{2}^{+} r_{1}^{+} & n_{1}^{+} r_{1}^{+}+n_{2}^{+} r_{2}^{+}
\end{array}\right)
\end{aligned}
$$

One has therefore

$$
\begin{gathered}
\int_{x_{1}^{-}-\frac{\varepsilon}{2}}^{x_{1}^{-}+\frac{\varepsilon}{2}} \mathbf{G}_{p p}^{-} r_{1}^{-} \mathrm{d} r_{1}^{-} \boldsymbol{\epsilon}^{-}\left(\mathbf{x}_{0}\right) \mathbf{e}_{1}^{-}+\mathbf{A}^{\top} \int_{x_{1}^{+}-\frac{\varepsilon}{2}}^{x_{1}^{+}+\frac{\varepsilon}{2}} \mathbf{G}_{p p}^{+} r_{1}^{+} \mathrm{d} r_{1}^{+} \mathbf{A} \boldsymbol{\epsilon}^{-}\left(\mathbf{x}_{0}\right) \mathbf{A}^{\top} \mathbf{e}_{1}^{+}= \\
{\left[\log \left\|\mathbf{r}^{-}\right\| \mathbf{L}_{p p}^{(1)}+\arctan \left(\frac{r_{1}^{-}}{x_{2}^{-}}\right) \mathbf{A}_{p p}^{(1)}+\frac{1}{r^{2}} \mathbf{S}_{p p}^{(1)}+\frac{1}{r^{4}} \mathbf{H}_{p p}^{(1)}\right]_{r_{1}^{-}=x_{1}^{-}-\frac{\varepsilon}{2}}^{r_{1}^{-}=x_{1}^{-}+\frac{\varepsilon}{2}} \boldsymbol{\epsilon}^{-}\left(\mathbf{x}_{0}\right) \mathbf{e}_{1}^{-}+} \\
{\left[\log \left\|\mathbf{r}^{+}\right\| \mathbf{A}^{\top} \mathbf{L}_{p p}^{(1)} \mathbf{A}+\arctan \left(\frac{r_{1}^{+}}{x_{2}^{+}\left(\mathbf{x}^{-}\right)}\right) \mathbf{A}^{\top} \mathbf{A}_{p p}^{(1)} \mathbf{A}+\right.} \\
\left.\frac{1}{r^{2}} \mathbf{A}^{\top} \mathbf{S}_{p p}^{(1)} \mathbf{A}+\frac{1}{r^{4}} \mathbf{A}^{\top} \mathbf{H}_{p p}^{(1)} \mathbf{A}\right]_{r_{1}^{+}=x_{1}^{+}\left(\mathbf{x}^{-}\right)-\frac{\varepsilon}{2}}^{r_{1}^{+}=x_{1}^{+}\left(\mathbf{x}^{-}\right)+\frac{\varepsilon}{2}} \boldsymbol{\epsilon}^{-}\left(\mathbf{x}_{0}\right) \mathbf{A}^{\top} \mathbf{e}_{1}^{+}
\end{gathered}
$$

The limit to the boundary $\Omega \ni \mathbf{x} \rightarrow \mathbf{x}_{0} \in \Gamma$ is taken by means of the norm $\left\|\mathbf{x}-\mathbf{x}_{0}\right\| \rightarrow 0$ and of a direction, selected through the angle $\theta$ as in figure 6 . Denote by:

$$
\begin{gathered}
\lim _{\mathbf{x} \rightarrow \mathbf{x}_{0}}\left\{\left[\frac{\mathbf{S}_{p p}^{(1)}}{r^{2}}\right]_{x_{1}^{-}-\frac{\varepsilon}{2}}^{x_{1}^{-}+\frac{\varepsilon}{2}} \boldsymbol{\epsilon}^{-}\left(\mathbf{x}_{0}\right) \mathbf{e}_{1}^{-}+\left[\frac{\mathbf{A}^{\top} \mathbf{S}_{p p}^{(1)} \mathbf{A}}{r^{2}}\right]_{x_{1}^{+}\left(\mathbf{x}^{-}\right)-\frac{\varepsilon}{2}}^{x_{1}^{+}\left(\mathbf{x}^{-}\right)+\frac{\varepsilon}{2}}\right. \\
\left.\boldsymbol{\epsilon}^{-}\left(\mathbf{x}_{0}\right) \mathbf{A}^{\top} \mathbf{e}_{1}^{+}\right\} \\
\lim _{\mathbf{x} \rightarrow \mathbf{x}_{0}}\left\{\left[\frac{\mathbf{H}_{p p}^{(1)}(\theta, \varphi, \mathbf{n}, \boldsymbol{\epsilon}, G, \nu)}{r^{4}}\right]_{x_{1}^{-}-\frac{\varepsilon}{2}}^{x_{1}^{-}+\frac{\varepsilon}{2}} \boldsymbol{\epsilon}^{-}\left(\mathbf{x}_{0}\right) \mathbf{e}_{1}^{-}+\left[\frac{\mathbf{A}^{\top} \mathbf{H}_{p p}^{(1)} \mathbf{A}}{r^{4}}\right]_{x_{1}^{+}\left(\mathbf{x}^{-}\right)-\frac{\varepsilon}{2}}^{x_{1}^{+}\left(\mathbf{x}^{-}\right)+\frac{\varepsilon}{2}} \boldsymbol{\epsilon}^{-}\left(\mathbf{x}_{0}\right) \mathbf{A}^{\top} \mathbf{e}_{1}^{+}\right\} \\
\lim _{\mathbf{x} \rightarrow \mathbf{x}_{0}}\left\{\left[\arctan \left(\frac{r_{1}^{+}}{x_{2}^{+}\left(\mathbf{x}^{-}\right)}\right) \mathbf{A}^{\top} \mathbf{A}_{p p}^{(1)} \mathbf{A}\right]_{x_{1}^{+}\left(\mathbf{x}^{-}\right)-\frac{\varepsilon}{2}}^{x_{1}^{+}\left(\mathbf{x}^{-}\right)+\frac{\varepsilon}{2}} \mathbf{\epsilon}_{0}^{-}\left(\mathbf{x}_{0}\right) \mathbf{A}^{\top} \mathbf{e}_{1}^{+}+\right. \\
{\left[\arctan \left(\frac{\left.r_{1}^{-}\right) \mathbf{A}_{p p}^{(1)}}{x_{2}^{-}}\right]_{x_{1}^{-}-\frac{\varepsilon}{2}}^{x_{1}^{-}+\frac{\varepsilon}{2}} \boldsymbol{\epsilon}^{-}\left(\mathbf{x}_{0}\right) \mathbf{e}_{1}^{-}\right\}} \\
=\mathbf{a}_{p p}^{1}(\theta, \varphi, \mathbf{n}, \boldsymbol{\epsilon}, G, \nu)
\end{gathered}
$$

where $\varphi$ is the angle between the normals $\mathbf{l}^{+}$and $\mathbf{l}^{-}$as in figure $6, \mathbf{n}$ is the selected direction, $G$ and $\nu$ are elastic properties of the body. ${ }^{2}$

\footnotetext{
${ }^{2}$ Note that the limits above do not exist, for they depend on the direction $\theta$ by which $\mathbf{x} \rightarrow \mathbf{x}_{0}$ (see figure 6 ); accordingly $\mathbf{s}_{p p}^{1}, \mathbf{h}_{p p}^{1}, \mathbf{a}_{p p}^{1}$ are but notations, not definitions.
} 
Functions $\mathbf{s}_{p p}^{1}, \mathbf{h}_{p p}^{1}$, and $\mathbf{a}_{p p}^{1}$, being independent on $\varepsilon$, act as free terms. It is worth noting that both $\mathbf{s}_{p p}^{1}$ and $\mathbf{h}_{p p}^{1}$ vanish at $\varphi=0$, as expected from the free term analysis on smooth boundaries. Further, at $\varphi=0$ it holds $\mathbf{e}_{1}^{+} \equiv \mathbf{e}_{1}^{-}$and

$$
\mathbf{a}_{p p}^{1}(\theta, \varphi, \mathbf{n}, \boldsymbol{\epsilon}, G, \nu)=\frac{G}{1-\nu}\left(\begin{array}{cc}
n_{1} & 0 \\
0 & 0
\end{array}\right) \boldsymbol{\epsilon}\left(\mathbf{x}_{0}\right) \mathbf{e}_{1}
$$

thus confirming the result of equation (13) of reference [1]. Finally, it holds:

$$
\begin{gathered}
\lim _{\mathbf{x} \rightarrow \mathbf{x}_{0}}\left\{\left[\log \left\|\mathbf{r}^{-}\right\| \mathbf{L}_{p p}^{(1)}\right]_{x_{1}^{-}-\frac{\varepsilon}{2}}^{x_{1}^{-}+\frac{\varepsilon}{2}} \boldsymbol{\epsilon}^{-}\left(\mathbf{x}_{0}\right) \mathbf{e}_{1}^{-}+\right. \\
\left.\left[\mathbf{A}^{\top} \mathbf{L}_{p p}^{(1)} \mathbf{A} \log \left\|\mathbf{r}^{+}\right\|\right]_{x_{1}^{+}\left(\mathbf{x}^{-}\right)-\frac{\varepsilon}{2}}^{x_{1}^{+}\left(\mathbf{x}^{-}\right)+\frac{\varepsilon}{2}} \boldsymbol{\epsilon}^{-}\left(\mathbf{x}_{0}\right) \mathbf{A}^{\top} \mathbf{e}_{1}^{+}\right\} \\
=\mathbf{l}_{p p}^{2}(\varphi, \mathbf{n}, \boldsymbol{\epsilon}, G, \nu) \log \|\varepsilon\|+\mathbf{l}_{p p}^{1}(\varphi, \mathbf{n}, \boldsymbol{\epsilon}, G, \nu) \lim _{\mathbf{x} \rightarrow \mathbf{x}_{0}} \log \left\|\mathbf{x}-\mathbf{x}_{0}\right\|
\end{gathered}
$$

with $\mathbf{l}_{p p}^{1}, \mathbf{l}_{p p}^{2}$ vanishing at $\varphi=0$. Note also that $\mathbf{l}_{p p}^{1}, \mathbf{l}_{p p}^{2}$ do not depend on $\theta$. By introducing Hadamard's finite part, it is possible to show that

$$
\begin{aligned}
f_{x_{1}^{-}-\frac{\varepsilon}{2}}^{x_{1}^{-}+\frac{\varepsilon}{2}} & \mathbf{G}_{p p}^{-} r_{1} \mathrm{~d} r_{1} \boldsymbol{\epsilon}^{-}\left(\mathbf{x}_{0}\right) \mathbf{e}_{1}^{-}+\mathbf{A}^{\top} f_{x_{1}^{+}-\frac{\varepsilon}{2}}^{x_{1}^{+}+\frac{\varepsilon}{2}} \mathbf{G}_{p p}^{+} r_{1} \mathrm{~d} r_{1} \mathbf{A} \boldsymbol{\epsilon}^{-}\left(\mathbf{x}_{0}\right) \mathbf{A}^{\top} \mathbf{e}_{1}^{+}= \\
& =\frac{G}{4 \pi(1-\nu)}\left[\mathbf{A}^{\top}\left(\begin{array}{cc}
n_{2}^{+} & n_{1}^{+} \\
n_{1}^{+} & n_{2}^{+}
\end{array}\right) \mathbf{A} \boldsymbol{\epsilon}^{-}\left(\mathbf{x}_{0}\right) \mathbf{A}^{\top} \mathbf{e}_{1}^{+}-\left(\begin{array}{cc}
n_{2}^{-} & n_{1}^{-} \\
n_{1}^{-} & n_{2}^{-}
\end{array}\right) \boldsymbol{\epsilon}^{-}\left(\mathbf{x}_{0}\right) \mathbf{e}_{1}^{-}\right] \log \|\varepsilon\| \\
& =\mathbf{l}_{p p}^{2}(\varphi, \mathbf{n}, \boldsymbol{\epsilon}, G, \nu) \log \|\varepsilon\|
\end{aligned}
$$

Accordingly,

$$
\begin{gathered}
\lim _{\mathbf{x} \rightarrow \mathbf{x}_{0}}\left\{\int_{x_{1}^{-}-\frac{\varepsilon}{2}}^{x_{1}^{-}+\frac{\varepsilon}{2}} \mathbf{G}_{p p}^{-} r_{1} \mathrm{~d} r_{1} \boldsymbol{\epsilon}^{-}\left(\mathbf{x}_{0}\right) \mathbf{e}_{1}^{-}+\mathbf{A}^{\top} \int_{x_{1}^{+}-\frac{\varepsilon}{2}}^{x_{1}^{+}+\frac{\varepsilon}{2}} \mathbf{G}_{p p}^{+} r_{1} \mathrm{~d} r_{1} \mathbf{A} \boldsymbol{\epsilon}^{-}\left(\mathbf{x}_{0}\right) \mathbf{A}^{\top} \mathbf{e}_{1}^{+}\right\}= \\
\mathbf{s}_{p p}^{1}+\mathbf{h}_{p p}^{1}+\mathbf{a}_{p p}^{1}+f_{x_{1}^{-}-\frac{\varepsilon}{2}}^{x_{1}^{-}+\frac{\varepsilon}{2}} \mathbf{G}_{p p}^{-} r_{1} \mathrm{~d} r_{1}+ \\
\mathbf{A}^{\top} f_{x_{1}^{+}-\frac{\varepsilon}{2}}^{x_{1}^{+}+\frac{\varepsilon}{2}} \mathbf{G}_{p p}^{+} r_{1} \mathrm{~d} r_{1} \mathbf{A}+\mathbf{l}_{p p}^{1}(\varphi, \mathbf{n}, \boldsymbol{\epsilon}, G, \nu) \lim _{\mathbf{x} \rightarrow \mathbf{x}_{0}} \log \left\|\mathbf{x}-\mathbf{x}_{0}\right\|
\end{gathered}
$$

The carried out analysis permits to conclude that, differently from the constant integral item, the unbounded terms do not cancel out in the limit process $\Omega \ni \mathbf{x} \rightarrow$ $\mathbf{x}_{0} \in \Gamma$. Identity (30) shows that the limit

$$
\lim _{\mathbf{x} \rightarrow \mathbf{x}_{0}} \int_{\Gamma_{\varepsilon}} \mathbf{G}_{p p}(\mathbf{x}-\mathbf{y} ; \mathbf{n}(\mathbf{x}) ; \mathbf{l}(\mathbf{y})) \mathbf{u}(\mathbf{y}) \mathrm{d} y_{1}
$$

is not bounded in the presence of corners and is equivalent to the HFP plus an unbounded logarithmic term. Furthermore the term:

$$
\mathbf{D}_{p p}\left(\mathbf{x}_{0}, \mathbf{n}\left(\mathbf{x}_{0}\right)\right) \stackrel{\text { def }}{=}-\left(\mathbf{s}_{p p}^{1}+\mathbf{h}_{p p}^{1}+\mathbf{a}_{p p}\right)^{1}
$$


which is expected to be the contribution of the hypersingular kernel to the freeterm $\mathbf{D}(\mathbf{x})$ in equation (2), is not well defined in the presence of corners. In fact, it depends on $\theta$ (i.e. the selected direction in the limit process).

Concluding, in the presence of corners two major differences with respect to the smooth boundary case arise. First, the boundary limit of the hypersingular kernel is not bounded. Further, its finite contribution (HFP plus free terms) depends on $\theta$. It will be proved later on in the paper that these two undesirable items cancel out in the sum with the strongly singular kernel boundary limit. In this sense, and only in this sense, the quantity (31) may be invoked as the contribution of the hypersingular kernel to the free term and the concept of HFP invoked.

All aforesaid evaluations have been made with the help of the computer code MATHEMATICA, release 4.0, because of the high complexity of the involved terms. In view of its interest, the sum $\mathbf{s}_{p p}^{1}+\mathbf{h}_{p p}^{1}+\mathbf{a}_{p p}^{1}$ is here extensively written after having expressed $\boldsymbol{\epsilon}^{-}\left(\mathbf{x}_{0}\right)$ in terms of $\boldsymbol{\sigma}^{-}\left(\mathbf{x}_{0}\right)$ by means of equation (18):

$$
\begin{aligned}
& \mathbf{s}_{p p}^{1}+\mathbf{h}_{p p}^{1}+\mathbf{a}_{p p}^{1}=\frac{|\sin (\theta)| \csc (\theta)}{16 \pi(-1+\nu) \operatorname{sgn}(\csc (\varphi+\theta))}\{ \\
& 2 \pi \cos (\varphi)\left(n_{1} \cos (\varphi)-n_{2} \sin (\varphi)\right) \\
& {\left[\left(\sigma_{11}+\sigma_{22}\right)(-1+2 \nu)+\left(-\sigma_{11}+\sigma_{22}\right) \cos (2 \varphi)+2 \sigma_{21} \sin (2 \varphi)\right]+} \\
& \operatorname{sgn}(\csc (\varphi+\theta) \sin (\theta)) \\
& {\left[4 n_{1} \pi\left(\sigma_{11}(-1+\nu)+\sigma_{22} \nu\right)+8 n_{1}\left(\sigma_{11}(-1+\nu)+\sigma_{22} \nu\right) \arctan (\cot (\theta))+\right.} \\
& 4 \arctan (\cot (\varphi+\theta)) \cos (\varphi) \quad\left(n_{1} \cos (\varphi)-n_{2} \sin (\varphi)\right) \\
& {\left[\sigma_{11}+\sigma_{22}-2\left(\sigma_{11}+\sigma_{22}\right) \nu+\left(\sigma_{11}-\sigma_{22}\right) \cos (2 \varphi)-2 \sigma_{21} \sin (2 \varphi)\right]+} \\
& \sin (\varphi) \\
& \left(\left(-4 n_{1} \sigma_{11}+8 n_{2} \sigma_{21}+4 n_{1} \sigma_{22}\right) \cos (3 \varphi)+\right. \\
& \left(13 n_{2} \sigma_{11}+20 n_{1} \sigma_{21}-3 n_{2} \sigma_{22}-10 n_{2} \sigma_{11} \nu-\right. \\
& 10 n_{2} \sigma_{22} \nu+8 n_{2} \sigma_{11} \cos (2 \varphi)+ \\
& +16 n_{1} \sigma_{21} \cos (2 \varphi)-8 n_{2} \sigma_{22} \cos (2 \varphi)- \\
& 4\left(n_{1} \sigma_{21}+n_{2}\left(\sigma_{11}-\sigma_{22}\right)\right) \cos (2 \theta)+ \\
& -2\left(2 n_{2} \sigma_{21}+n_{1}\left(-3 \sigma_{11}+\sigma_{22}+2\left(\sigma_{11}+\sigma_{22}\right) \nu\right)\right) \sin (2 \theta)+ \\
& \left.+\left(\sigma_{11}+\sigma_{22}\right)(-1+2 \nu)\left(n_{2} \cos (4 \theta)-n_{1} \sin (4 \theta)\right)\right) \sin (\varphi)+ \\
& \left(8 n_{2} \sigma_{21}+n_{1}\left(-11 \sigma_{11}+\sigma_{22}+10\left(\sigma_{11}+\sigma_{22}\right) \nu\right)-\right. \\
& 4\left(2 n_{2} \sigma_{21}+n_{1}\left(\sigma_{22}-\left(\sigma_{11}+\sigma_{22}\right) \nu\right)\right) \cos (2 \theta)+ \\
& \left(\sigma_{11}+\sigma_{22}\right)(-1+2 \nu)\left(n_{1} \cos (4 \theta)+n_{2} \sin (4 \theta)\right)+ \\
& \left.\left.\left.2\left(n_{2} \sigma_{11}+4 n_{1} \sigma_{21}-n_{2} \sigma_{22}\right) \sin (2 \theta)\right) \quad \cos (\varphi)\right)\right] \text {, }
\end{aligned}
$$




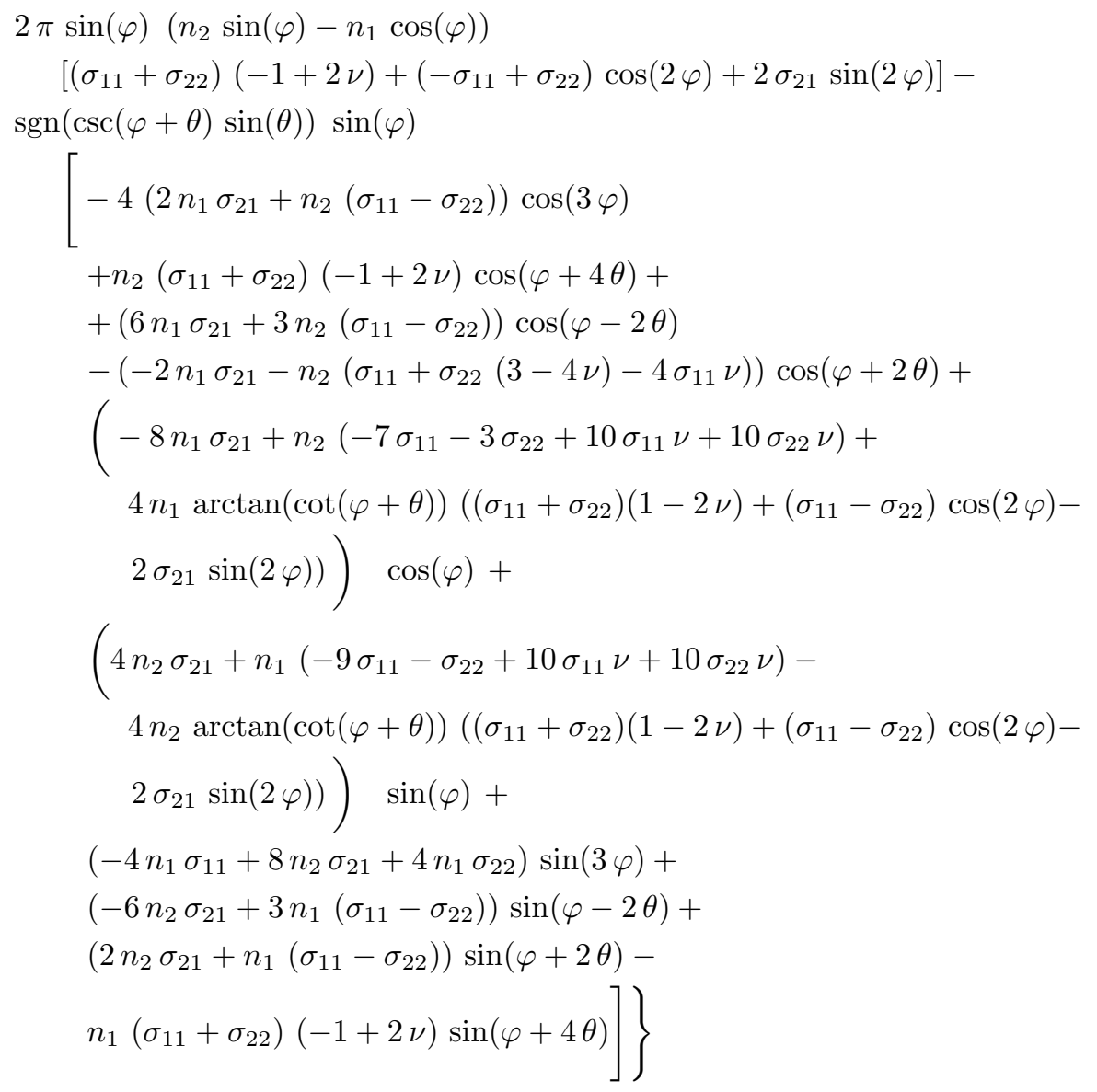

\section{Appendix 2 - Strongly singular kernel integration at a corner.}

This appendix focuses on the mathematical passages required by the free term analysis of the strongly singular integral (15). By assuming $\mathbf{x} \in \Omega$ and in view of identity (16), consider the two integrals of $(15)$ in the local references $\mathcal{L}^{+}, \mathcal{L}^{-}$:

$$
\int_{\Gamma_{\varepsilon}^{-}} \mathbf{G}_{p u}^{-} \mathrm{d} \Gamma=\int_{-\varepsilon}^{0} \mathbf{G}_{p u}^{-} \mathrm{d} y_{1}+O(\varepsilon) \quad \int_{\Gamma_{\varepsilon}^{+}} \mathbf{G}_{p u}^{+} \mathrm{d} \Gamma=\int_{0}^{\varepsilon} \mathbf{G}_{p u}^{+} \mathrm{d} y_{1}+O(\varepsilon)
$$

In order to sum all contributions, the same coordinate system must be set. With reference to figure 5 the matrix

$$
\mathbf{A}=\left(\begin{array}{cc}
\cos \varphi & -\sin \varphi \\
\sin \varphi & \cos \varphi
\end{array}\right)
$$


governs the reference change between $\mathcal{L}^{-}$and $\mathcal{L}^{+}$: accordingly $X^{-}=\mathbf{A}^{\top} X^{+} \mathbf{A}$ for any tensor $X^{+}$in the local reference $\mathcal{L}^{+}$and $x^{-}=\mathbf{A}^{\top} x^{+}$for any vector $x^{+}$in the local reference $\mathcal{L}^{+}$. For being

$\mathbf{p}^{-}=\mathbf{A}^{\top} \mathbf{p}^{+} \sim \mathbf{A}^{\top} \mathbf{G}_{p u}^{+} \mathbf{p}^{+}\left(\mathbf{x}_{0}\right)=\mathbf{A}^{\top} \mathbf{G}_{p u}^{+} \mathbf{A} \mathbf{A}^{\top} \mathbf{p}^{+}\left(\mathbf{x}_{0}\right)=\mathbf{A}^{\top} \mathbf{G}_{p u}^{+} \mathbf{A} \boldsymbol{\sigma}^{-}\left(\mathbf{x}_{0}\right) \mathbf{A}^{\top} \mathbf{l}^{+}\left(\mathbf{x}_{0}\right)$

it comes out that integral (15) holds:

$$
\int_{-\varepsilon}^{0} \mathbf{G}_{p u}^{-} \mathrm{d} y_{1} \boldsymbol{\sigma}^{-}\left(\mathbf{x}_{0}\right) \mathbf{e}_{2}^{-}+\mathbf{A}^{\top} \int_{0}^{\varepsilon} \mathbf{G}_{p u}^{+} \mathrm{d} y_{1} \mathbf{A} \boldsymbol{\sigma}^{-}\left(\mathbf{x}_{0}\right) \mathbf{A}^{\top} \mathbf{l}^{+}
$$

in reference $\mathcal{L}^{-}$. The integral

$$
\int_{0}^{\varepsilon} \mathbf{G}_{p p}^{+} \mathrm{d} y_{1}^{+}=\log \left\|\mathbf{r}^{+}\right\| \mathbf{L}_{p u}+\arctan \left(\frac{r_{1}^{+}}{x_{2}^{+}}\right) \mathbf{A}_{p u}+\left.\frac{1}{r^{2}} \mathbf{S}_{p u}\right|_{r_{1}^{+}=x_{1}^{+}-\frac{\varepsilon}{2}} ^{r_{1}^{+}=x_{1}^{+}+\frac{\varepsilon}{2}} \quad \mathbf{x} \in \Omega
$$

has been solved in [2] by means of the variable change $\mathbf{r}^{+}=\mathbf{x}^{+}-\mathbf{y}^{+}$; matrices $\mathbf{L}_{p u}, \mathbf{A}_{p u}$, and $\mathbf{S}_{p u}$ follow in the local reference $\mathcal{L}^{+}$:

$$
\begin{aligned}
\mathbf{L}_{p u} & =\frac{1}{4 \pi(1-\nu)}\left(\begin{array}{cc}
n_{1}(-3+2 \nu) & n_{2}(-1+2 \nu) \\
n_{2}(1-2 \nu) & n_{1}(-1+2 \nu)
\end{array}\right) \\
\mathbf{A}_{p u} & =\frac{1}{2 \pi(1-\nu)}\left(\begin{array}{cc}
n_{2}(-1+\nu) & -n_{1} \nu \\
n_{1}(-1+\nu) & n_{2}(-1+\nu)
\end{array}\right) \\
\mathbf{S}_{p u} & =\frac{r_{2}}{4 \pi(1-\nu)}\left(\begin{array}{cc}
n_{2} r_{1}-n_{1} r_{2} & n_{1} r_{1}+n_{2} r_{2} \\
n_{1} r_{1}+n_{2} r_{2} & -n_{2} r_{1}+n_{1} r_{2}
\end{array}\right)
\end{aligned}
$$

One has therefore when $\mathbf{x} \in \Omega$

$$
\begin{aligned}
& \int_{-\varepsilon}^{0} \mathbf{G}_{p u}^{-} \mathrm{d} y_{1} \boldsymbol{\sigma}^{-}\left(\mathbf{x}_{0}\right) \mathbf{e}_{2}^{-}+\mathbf{A}^{\top} \int_{0}^{\varepsilon} \mathbf{G}_{p u}^{+} \mathrm{d} y_{1} \mathbf{A} \boldsymbol{\sigma}^{-}\left(\mathbf{x}_{0}\right) \mathbf{A}^{\top} \mathbf{l}^{+}= \\
& {\left[\log \left\|\mathbf{r}^{-}\right\| \mathbf{L}_{p u}+\arctan \left(\frac{r_{1}^{-}}{x_{2}^{-}}\right) \mathbf{A}_{p u}+\frac{1}{r^{2}} \mathbf{S}_{p u}\right]_{r_{1}^{-}=x_{1}^{-}-\frac{\varepsilon}{2}}^{\sigma_{1}^{-}=x_{1}^{-}+\frac{\varepsilon}{2}}\left(\mathbf{x}_{0}\right) \mathbf{e}_{2}^{-}+} \\
& {\left[\log \left\|\mathbf{r}^{+}\right\| \mathbf{A}^{\top} \mathbf{L}_{p u} \mathbf{A}+\arctan \left(\frac{r_{1}^{+}}{x_{2}^{+}\left(\mathbf{x}^{-}\right)}\right) \mathbf{A}^{\top} \mathbf{A}_{p u} \mathbf{A}+\frac{1}{r^{2}} \mathbf{A}^{\top} \mathbf{S}_{p u} \mathbf{A}\right]_{r_{1}^{+}=x_{1}^{+}\left(\mathbf{x}^{-}\right)-\frac{\varepsilon}{2}}^{r_{1}^{+}=x_{1}^{+}\left(\mathbf{x}^{-}\right)+\frac{\varepsilon}{2}}} \\
& \boldsymbol{\sigma}^{-}\left(\mathbf{x}_{0}\right) \mathbf{A}^{\top} \mathbf{l}^{+}
\end{aligned}
$$

The limit to the boundary $\Omega \ni \mathbf{x} \rightarrow \mathbf{x}_{0} \in \Gamma$ is taken by means of the norm $\left\|\mathbf{x}-\mathbf{x}_{0}\right\| \rightarrow 0$ and of a direction, selected through the angle $\theta$ as in figure 6 . Denote with:

$$
\begin{gathered}
\lim _{\mathbf{x} \rightarrow \mathbf{x}_{0}}\left\{\left[\frac{\mathbf{S}_{p u}}{r^{2}}\right]_{x_{1}^{-}-\frac{\varepsilon}{2}}^{x_{1}^{-}+\frac{\varepsilon}{2}} \boldsymbol{\sigma}^{-}\left(\mathbf{x}_{0}\right) \mathbf{e}_{2}^{-}+\right. \\
\left.=\left[\frac{\mathbf{A}^{\top} \mathbf{S}_{p u} \mathbf{A}}{r^{2}}\right]_{x_{1}^{+}\left(\mathbf{x}^{-}\right)-\frac{\varepsilon}{2}}^{x_{1}^{+}\left(\mathbf{x}^{-}\right)+\frac{\varepsilon}{2}} \boldsymbol{\sigma}^{-}\left(\mathbf{x}_{0}\right) \mathbf{A}^{\top} \mathbf{l}^{+}\right\} \\
=\mathbf{s}_{p u}(\theta, \varphi, \mathbf{n}, \boldsymbol{\sigma}, \nu)
\end{gathered}
$$




$$
\begin{array}{r}
\lim _{\mathbf{x} \rightarrow \mathbf{x}_{0}}\left\{\left[\arctan \left(\frac{r_{1}^{+}}{x_{2}^{+}\left(\mathbf{x}^{-}\right)}\right) \mathbf{A}^{\top} \mathbf{A}_{p u} \mathbf{A}\right]_{x_{1}^{+}\left(\mathbf{x}^{-}\right)-\frac{\varepsilon}{2}}^{x_{1}^{+}\left(\mathbf{x}^{-}\right)+\frac{\varepsilon}{2}} \boldsymbol{\sigma}^{-}\left(\mathbf{x}_{0}\right) \mathbf{A}^{\top} \mathbf{l}^{+}+\right. \\
\left.\left[\arctan \left(\frac{r_{1}^{-}}{x_{2}^{-}}\right) \mathbf{A}_{p u}\right]_{x_{1}^{-}-\frac{\varepsilon}{2}}^{x_{1}^{-}+\frac{\varepsilon}{2}} \boldsymbol{\sigma}^{-}\left(\mathbf{x}_{0}\right) \mathbf{e}_{2}^{-}\right\}=\mathbf{a}_{p u}(\theta, \varphi, \mathbf{n}, \boldsymbol{\sigma}, \nu)
\end{array}
$$

where $\varphi$ is the angle between the normals $\mathbf{l}^{+}$and $\mathbf{l}^{-} \equiv \mathbf{e}_{2}^{-}$as in figure $6, \mathbf{n}$ is the selected direction, and $\nu$ is Poisson's coefficient of the body. ${ }^{3}$

Functions $\mathbf{s}_{p u}$ and $\mathbf{a}_{p u}$, being independent on $\varepsilon$, act as free terms. It is worth noting that $\mathbf{s}_{p u}$ vanishes at $\varphi=0$ for any $\theta$, as expected from the free term analysis on smooth boundaries. Further, at $\varphi=0$ it holds $\mathbf{l}^{+} \equiv \mathbf{e}_{2}^{-}$and

$$
\mathbf{a}_{p u}(\theta, \varphi, \mathbf{n}, \boldsymbol{\sigma}, \nu)=\frac{1}{2} \frac{1}{1-\nu}\left(\begin{array}{cc}
(1-\nu) n_{2} & \nu n_{1} \\
(1-\nu) n_{1} & (1-\nu) n_{2}
\end{array}\right) \boldsymbol{\sigma}\left(\mathbf{x}_{0}\right) \mathbf{e}_{2}^{-}
$$

By comparison with equation (27) of reference [1] the role of $\mathbf{a}_{p u}$ as a free term is confirmed. Finally, it holds:

$$
\begin{gathered}
\lim _{\mathbf{x} \rightarrow \mathbf{x}_{0}}\left\{\left[\log \left\|\mathbf{r}^{-}\right\| \mathbf{L}_{p u}\right]_{x_{1}^{-}-\frac{\varepsilon}{2}}^{x_{-}^{-}+\frac{\varepsilon}{2}} \boldsymbol{\sigma}^{-}\left(\mathbf{x}_{0}\right) \mathbf{e}_{2}^{-}+\left[\mathbf{A}^{\top} \mathbf{L}_{p u} \mathbf{A} \log \left\|\mathbf{r}^{+}\right\|\right]_{x_{1}^{+}\left(\mathbf{x}^{-}\right)-\frac{\varepsilon}{2}}^{x^{+}\left(\mathbf{x}^{-}\right)+\frac{\varepsilon}{2}} \boldsymbol{\sigma}^{-}\left(\mathbf{x}_{0}\right) \mathbf{A}^{\top} \mathbf{l}^{+}\right\} \\
=\mathbf{l}_{p u}(\varphi, \mathbf{n}, \boldsymbol{\sigma}, \nu) \log \|\varepsilon\|-\mathbf{l}_{p p}^{1}(\varphi, \mathbf{n}, \boldsymbol{\epsilon}(\boldsymbol{\sigma}), G, \nu) \lim _{\mathbf{x} \rightarrow \mathbf{x}_{0}} \log \left\|\mathbf{x}-\mathbf{x}_{0}\right\|
\end{gathered}
$$

with $\mathbf{l}_{p p}^{1}$ defined in appendix 1 . Note that $\mathbf{l}_{p p}^{1}$ and $\mathbf{l}_{p u}$ vanish at $\varphi=0$ and do not depend on $\theta$. By introducing the HFP,

$$
\begin{gathered}
f_{-\varepsilon}^{0} \mathbf{G}_{p u}^{-} \mathrm{d} y_{1} \boldsymbol{\sigma}^{-}\left(\mathbf{x}_{0}\right) \mathbf{e}_{2}^{-}+\mathbf{A}^{\top} f_{0}^{\varepsilon} \mathbf{G}_{p u}^{+} \mathrm{d} y_{1} \mathbf{A} \boldsymbol{\sigma}^{-}\left(\mathbf{x}_{0}\right) \mathbf{A}^{\top} \mathbf{l}^{+}= \\
\mathbf{A}^{\top} f_{-\varepsilon}^{0} \mathbf{G}_{p u}^{+} \mathrm{d} r_{1} \mathbf{A} \boldsymbol{\sigma}^{-}\left(\mathbf{x}_{0}\right) \mathbf{A}^{\top} \mathbf{l}^{+}+f_{0}^{\varepsilon} \mathbf{G}_{p u}^{-} \mathrm{d} r_{1} \boldsymbol{\sigma}^{-}\left(\mathbf{x}_{0}\right) \mathbf{e}_{2}^{-}= \\
\frac{1}{4 \pi(1-\nu)}\left[\left(\begin{array}{cc}
n_{1}^{-}(-3+2 \nu) & n_{2}^{-}(-1+2 \nu) \\
n_{2}^{-}(1-2 \nu) & n_{1}^{-}(-1+2 \nu)
\end{array}\right) \boldsymbol{\sigma}^{-}\left(\mathbf{x}_{0}\right) \mathbf{e}_{2}^{-}+\right. \\
\left.-\mathbf{A}^{\top}\left(\begin{array}{cc}
n_{1}^{+}(-3+2 \nu) & n_{2}^{+}(-1+2 \nu) \\
n_{2}^{+}(1-2 \nu) & n_{1}^{+}(-1+2 \nu)
\end{array}\right) \mathbf{A} \boldsymbol{\sigma}^{-}\left(\mathbf{x}_{0}\right) \mathbf{A}^{\top} \mathbf{l}^{+}\right] \log \|\varepsilon\|
\end{gathered}
$$

it is possible to show, by direct integration, that

$$
f_{-\varepsilon}^{0} \mathbf{G}_{p u}^{-} \mathrm{d} y_{1} \boldsymbol{\sigma}^{-}\left(\mathbf{x}_{0}\right) \mathbf{e}_{2}^{-}+\mathbf{A}^{\top} f_{0}^{\varepsilon} \mathbf{G}_{p u}^{+} \mathrm{d} y_{1} \mathbf{A} \boldsymbol{\sigma}^{-}\left(\mathbf{x}_{0}\right) \mathbf{A}^{\top} \mathbf{l}^{+}=\mathbf{l}_{p u}(\varphi, \mathbf{n}, \boldsymbol{\sigma}, \nu) \log \|\varepsilon\|
$$

Accordingly,

$$
\lim _{\mathbf{x} \rightarrow \mathbf{x}_{0}}\left\{\int_{-\varepsilon}^{0} \mathbf{G}_{p u}^{-} \mathrm{d} y_{1} \boldsymbol{\sigma}^{-}\left(\mathbf{x}_{0}\right) \mathbf{e}_{2}^{-}+\mathbf{A}^{\top} \int_{0}^{\varepsilon} \mathbf{G}_{p u}^{+} \mathrm{d} y_{1} \mathbf{A} \boldsymbol{\sigma}^{-}\left(\mathbf{x}_{0}\right) \mathbf{A}^{\top} \mathbf{l}^{+}\right\}=
$$

\footnotetext{
${ }^{3}$ Note that the limits above do not exist, for they depend on the direction $\theta$ by which $\mathbf{x} \rightarrow \mathbf{x}_{0}$ (see figure 6); accordingly $\mathbf{s}_{p u}, \mathbf{a}_{p u}$ are but notations, not definitions.
} 


$$
\begin{gathered}
f_{-\varepsilon}^{0} \mathbf{G}_{p u}^{-} \mathrm{d} y_{1} \boldsymbol{\sigma}^{-}\left(\mathbf{x}_{0}\right) \mathbf{e}_{2}^{-}+\mathbf{A}^{\top} f_{0}^{\varepsilon} \mathbf{G}_{p u}^{+} \mathrm{d} y_{1} \mathbf{A} \boldsymbol{\sigma}^{-}\left(\mathbf{x}_{0}\right) \mathbf{A}^{\top} \mathbf{l}^{+}+ \\
\mathbf{s}_{p u}+\mathbf{a}_{p u}-\mathbf{l}_{p p}^{1}(\varphi, \mathbf{n}, \boldsymbol{\epsilon}(\boldsymbol{\sigma}), G, \nu) \lim _{\mathbf{x} \rightarrow \mathbf{x}_{0}} \log \left\|\mathbf{x}-\mathbf{x}_{0}\right\|
\end{gathered}
$$

The limit (32) is a fundamental result for the free term analysis: it shows that, in the presence of corners, the strongly singular integral produces unbounded terms that do not cancel out in the limit process $\Omega \ni \mathbf{x} \rightarrow \mathbf{x}_{0} \in \Gamma$. The limit (32) equals Hadamard's finite part, plus an unbounded logarithmic term and the following factor

$$
\mathbf{D}_{p u}\left(\mathbf{x}_{0}, \mathbf{n}\left(\mathbf{x}_{0}\right)\right) \stackrel{\text { def }}{=} \mathbf{s}_{p u}+\mathbf{a}_{p u}
$$

which plays the role of free term, being independent on $\varepsilon$. Such a term depends on the selected direction in the limit process, and cannot be defined as a result of a limit process (32).

In conclusion, the strongly singular kernel shows the same peculiarities of the hypersingular kernel with regard to the free term analysis. As seen in section 4, these facts provide a fundamental property for the global problem.

The sum $\mathbf{s}_{p u}+\mathbf{a}_{p u}$ is here extensively written for its interest in what follows.

$$
\begin{aligned}
& \mathbf{s}_{p u}+\mathbf{a}_{p u}=\frac{1}{32 \pi(-1+\nu)}\{ \\
& -4 \sin (\varphi)\left(2\left(n_{2} \sigma_{21}+n_{1} \sigma_{22}\right) \cos (\varphi)+2\left[\left(n_{2} \sigma_{11}+2 n_{1} \sigma_{21}-n_{2} \sigma_{22}\right) \cos (2 \varphi-\theta)+\right.\right. \\
& \left.\left.+\left(n_{1} \sigma_{11}-2 n_{2} \sigma_{21}-n_{1} \sigma_{22}\right) \sin (2 \varphi-\theta)\right] \sin (\varphi+\theta)\right)+ \\
& +4 \cos (2 \varphi)\left(n_{1} \sigma_{21}-n_{2} \sigma_{22}+2 n_{1}\left(\sigma_{11}(-1+\nu)+\sigma_{22} \nu\right) \arctan (\cot (\varphi+\theta))+\right. \\
& \left.-\frac{n_{1} \pi\left(\sigma_{11}(-1+\nu)+\sigma_{22} \nu\right)}{\operatorname{sgn}(\csc (\varphi+\theta) \sin (\theta))}\right)+ \\
& -2 \sin (2 \varphi)\left(-2 n_{1} \sigma_{21}+n_{2}\left(\sigma_{11}+\sigma_{22}\right)(-1+2 \nu)\right) \\
& (2 \arctan (\cot (\varphi+\theta))-\pi|\csc (\varphi+\theta)||\sin (\theta)| \csc (\theta) \sin (\varphi+\theta))+ \\
& +\cos (3 \varphi)\left(-4 n_{1} \sigma_{11}+8 n_{2} \sigma_{21}+4 n_{1} \sigma_{22}\right)+ \\
& +\cos (4 \varphi)\left(2 n_{2} \sigma_{21}+n_{1}\left(-\sigma_{11}+\sigma_{22}\right)\right) \\
& (2 \arctan (\cot (\varphi+\theta))-\pi|\csc (\varphi+\theta)||\sin (\theta)| \csc (\theta) \sin (\varphi+\theta))+ \\
& +\sin (4 \varphi)\left(2 n_{1} \sigma_{21}+n_{2}\left(\sigma_{11}-\sigma_{22}\right)\right) \\
& (2 \arctan (\cot (\varphi+\theta))-\pi|\csc (\varphi+\theta)||\sin (\theta)| \csc (\theta) \sin (\varphi+\theta))+ \\
& -16\left(n_{1} \sigma_{22} \nu+n_{2}\left(\sigma_{21}-\sigma_{21} \nu\right)\right) \arctan (\cot (\theta))+ \\
& -2\left(2 n_{2} \sigma_{21}(-3+4 \nu)+n_{1}\left(\sigma_{22}-4 \sigma_{22} \nu+\sigma_{11}(-5+4 \nu)\right)\right) \arctan (\cot (\varphi+\theta))+ \\
& -4\left(-\left(n_{2}\left(\sigma_{22}+2 \pi \sigma_{21}(-1+\nu)\right)\right)+n_{1}\left(\sigma_{21}+2 \pi \sigma_{22} \nu\right)\right)+ \\
& +\pi\left(2 n_{2} \sigma_{21}(-3+4 \nu)+n_{1}\left(-5 \sigma_{11}+\sigma_{22}+4 \sigma_{11} \nu-4 \sigma_{22} \nu\right)\right) \\
& |\csc (\varphi+\theta)||\sin (\theta)| \csc (\theta) \sin (\varphi+\theta), \\
& -4 \sin (\varphi)\left(2 \left[\left(-2 n_{2} \sigma_{21}+n_{1}\left(\sigma_{11}-\sigma_{22}\right)\right) \cos (2 \varphi-\theta)+\right.\right.
\end{aligned}
$$




$$
\begin{aligned}
& \left.\left.\quad+\left(-2 n_{1} \sigma_{21}+n_{2}\left(-\sigma_{11}+\sigma_{22}\right)\right) \sin (2 \varphi-\theta)\right] \sin (\varphi+\theta)\right)+ \\
& +\sin (2 \varphi) \\
& \quad\left(4\left(2 n_{2} \sigma_{21}+n_{1}\left(\sigma_{11}+\sigma_{22}\right)(1-2 \nu)\right) \arctan (\cot (\varphi+\theta))+\right. \\
& \quad+\frac{-2 n_{1} \pi \sigma_{11}-4 n_{2} \pi \sigma_{21}+4 n_{1} \pi\left(\sigma_{11}+\sigma_{22}\right) \nu}{\operatorname{sgn}(\csc (\varphi+\theta) \sin (\theta))}+ \\
& \left.\quad-2\left(2 n_{1} \sigma_{21}-2 n_{2} \sigma_{22}+n_{1} \pi \sigma_{22}|\csc (\varphi+\theta)||\sin (\theta)| \csc (\theta) \sin (\varphi+\theta)\right)\right)+ \\
& +\cos (2 \varphi) \\
& \quad\left(-4\left(n_{2} \sigma_{21}+n_{1} \sigma_{22}+2 n_{2}\left(\sigma_{22}(-1+\nu)+\sigma_{11} \nu\right) \arctan (\cot (\varphi+\theta))\right)+\right. \\
& \quad+\frac{4 n_{2} \pi\left(\sigma_{22}(-1+\nu)+\sigma_{11} \nu\right)}{\operatorname{sgn}(\csc (\varphi+\theta) \sin (\theta))+} \\
& +\cos (4 \varphi)\left(2 n_{1} \sigma_{21}+n_{2}\left(\sigma_{11}-\sigma_{22}\right)\right) \\
& \quad(2 \arctan (\cot (\varphi+\theta))-\pi|\csc (\varphi+\theta)||\sin (\theta)| \csc (\theta) \sin (\varphi+\theta))+ \\
& +\sin (4 \varphi)\left(2 n_{2} \sigma_{21}+n_{1}\left(-\sigma_{11}+\sigma_{22}\right)\right) \\
& \quad(-2 \arctan (\cot (\varphi+\theta))+\pi|\csc (\varphi+\theta)||\sin (\theta)| \csc (\theta) \sin (\varphi+\theta))+ \\
& +16\left(n_{1} \sigma_{21}+n_{2} \sigma_{22}\right)(-1+\nu) \arctan (\cot (\theta))+ \\
& -2\left(2 n_{1} \sigma_{21}(-3+4 \nu)+n_{2}\left(\sigma_{11}-4 \sigma_{11} \nu+\sigma_{22}(-5+4 \nu)\right)\right) \arctan (\cot (\varphi+\theta))+ \\
& +4\left(n_{1}\left(\sigma_{22}+2 \pi \sigma_{21}(-1+\nu)\right)+n_{2}\left(\sigma_{21}+2 \pi \sigma_{22}(-1+\nu)\right)\right)+ \\
& +\pi\left(2 n_{1} \sigma_{21}(-3+4 \nu)+n_{2}\left(\sigma_{11}-5 \sigma_{22}-4 \sigma_{11} \nu+4 \sigma_{22} \nu\right)\right) \\
& \quad|\csc (\varphi+\theta)||\sin (\theta)| \csc (\theta) \sin (\varphi+\theta)\} \\
& +
\end{aligned}
$$

